\title{
Assessment of Effective Thermal Conductivity in U-Mo Metallic Fuels with Distributed Gas Bubbles
}

\author{
Shenyang Hu, Andrew M. Casella, Curt A Lavender, David J. Senor, and Douglas E. Burkes \\ Pacific Northwest National Laboratory, P. O. Box 999, Richland, WA 99352
}

\begin{abstract}
This work presents a numerical method to assess the relative impact of various microstructural features including grain sizes, nanometer scale intragranular gas bubbles, and larger intergranular gas bubbles in irradiated U-Mo metallic fuels on the effective thermal conductivity. A phasefield model was employed to construct a three-dimensional polycrystalline U-Mo fuel alloy with a given crystal morphology and gas bubble microstructures. An effective thermal conductivity "concept" was taken to capture the effect of polycrystalline structures and gas bubble microstructures with significant size differences on the thermal conductivity. The thermal conductivity of inhomogeneous materials was calculated by solving the heat transport equation. The obtained results are in reasonably good agreement with experimental measurements made on irradiated U-Mo fuel samples containing similar microstructural features. The developed method can be used to predict the thermal conductivity degradation in operating nuclear fuels if the evolution of microstructures is known during operation of the fuel.
\end{abstract}

\section{Introduction}

U-Mo alloy is a promising candidate for low enriched uranium fuels with high U loading. The gamma $\mathrm{U}$ phase is retained by Mo in solid solution that possesses acceptable irradiation stability, mechanical properties, and corrosion resistance, and is formed over a wide range of Mo concentration [1,2]. However, the formation and growth of fission gas bubbles in irradiated nuclear fuels is of technical importance because it may impact the thermo-mechanical properties, hence, fuel performance. Figure 1 shows the gas bubble structures in U-10Mo fuels at increasing burnups. TEM and SEM results indicate that dense intragranular fission gas bubbles are very small with a diameter of $2 \mathrm{~nm}$ and a bubble spacing of $6 \sim 7 \mathrm{~nm}$ [3]. Intergranular gas bubbles are larger than the intragranular gas bubbles with a size of hundreds of nanometers when the fission density exceeds $4 \times 10^{21} \mathrm{f} / \mathrm{m}^{3}$ [4]. As the fission density is increased further, recrystallization of the alloy occurs where U-Mo grains become finer. Gas bubbles coalesce at the recrystallization zone while intragranular gas bubbles disappear. The thermal conductivity of the gas phase (Xe and $\mathrm{Kr}$ ) is about $0.08 \mathrm{~W} / \mathrm{mK}$ that is significantly less than that of U-Mo (around $15 \mathrm{~W} / \mathrm{mK}$ at 
$\left.150^{\circ} \mathrm{C}\right)[5,6]$. As a consequence, the formation of gas bubbles might greatly impact the thermal conductivity of the metal fuel. On the other hand, different material processes such as heat treatment, hot and cold rolling, etc. are used to optimize the microstructure and performance of U-Mo metal fuels. It is commonly accepted that gas bubbles prefer to nucleate and grow on the grain boundaries. So grain morphology including grain size distribution, grain aspect ratio and average grain size may affect the gas bubble structure, hence, the effective thermal conductivity. Therefore, it is important to develop a method to assess the effect of initial microstructure and gas bubble microstructures on thermal conductivity for guiding the material process to obtain desired initial microstructures and fuel performance. The goal of such a method is not necessarily to predict absolute thermal conductivity values, but rather to assess the relative impact of various microstructural features that can be engineered during fabrication and influenced during irradiation.

A number of analytical and emperical models have been proposed to describe the effect of microstrucral heterogeneity such as pores, gas filled pores, and second phase preciitates on thermal conductivity. Expressions for the alteration of conductivity by dilute distributions of spherical pores are well known and were first derived by Maxwell[7]. Based on one directional heat flux assumption, Kampf and Peddicord [8-11] developed analytical porosity correlations of thermal conductivity for structures with distributed cubic and spherical pores. These theoretical models usually are valid to structures where the pore has regular shape, and the pore distribution is sufficiently dilute that perturbations produced by pores do not interfere with one aother. Bauer [12] generalizes Maxwell's results to random but uniform pore distribtions of any shape, conductivity, or concentration. Numerical methods have also been used to calculate the effect of heterogeneous microstructures on thermal conductivity. Yun el at. [13] used COMSOL multiphysics simulation platform to model heat transport in metallic U-10Zr fuel with distributed spherical pores. A finite element method (FEM) was used to calculate the effective change in grain boundary kapitza resistance due to the presence of elliptic intergranular gas bubbles in a bicrystal $\mathrm{UO}_{2}$ nuclear fuels [14]. Very recently, Teague et al[15] used FEM to assess the effect of defects including porosity, precipitates, and fission product layer on thermal conductivity in irradiated mixed oxide (MOX) fuels by directly using the three-dimensional microstructures from advanced 3D microstructure reconstruction techniques. However, the microstructures in irradiated nuclear fuels are very complicated. For example, gas bubbles in irradiated nuclear fuels have strong inhomogeneous distribution which depends on the grain morphology. The intra- and inter-granular gas bubbles have not only huge size differences, but also large morphological differences. Therefore, accurate construction of complicated microstructures is challenging, but is crucial to assess the effect of microstructures on thermal conductivity in irradiated nuclear fuels. The phase-field approach has been successfully used to model the evolution of three-dimensional microstructures [16, 17]. With accurate thermal dynamic properties, such as free energies of different phases and interfacial energies, the phase-field simulations can predict the evolving microstructures observed during post-irradiation examination. The intragranular gas bubbles usually have a spherical shape while intergranular 
gas bubbles are usually lens shaped to satisfy the equilibrium condition of interface tension at three phase interface. With the phase-field method, the morphology of intergranular gas bubbles can be captured that is important for the accurate calculation of effective thermal conductivity. The phase-field model has been used to generate the microstructures for assessing the effect of microstructures on the thermal conductivity. For instance, $\mathrm{Hu}$ et al [18, 19] used phase-field model to study the effect of evolving gas bubbles on the thermal conductivity in polycrystalline nuclear fuels. Millett et al [20-22] has investigated the effect of voids, intergranular gas bubbles, and irradiation conditions on thermal conductivity in polycrystals. But all these simulations were carried out in two demensions. In very recent work, Teague et al[15] claimed that a phase-field model is used to relax the three dimensional microstructure before they calculated the thermal conductivity. This work will present a numerical method to assess the effect of three dimensional polycrystalline microstructure and gas bubble microstructures on thermal conductivity. A multicomponent and multiphase field model was developed to simulate the grain and gas bubble evolution and generate the heterogeneous three-dimensional microstructure for given polycrystalline and gas bubble microstructures. The relevant thermal conductivities were assigned to the interface, matrix and gas phase, respectively. The effective thermal conductivity was calculated by solving the heat transport equation in a polycrystalline material with heterogeneous thermal conductivity. Since the intergranular gas bubble is about two orders of magnitude greater than that of intragranular gas bubbles, and the average grain size of different U-Mo fuels may also vary by an order of magnitude, it is challenging to capture the entire

microstructure in a single simulation cell. Therefore, the effective thermal conductivity of lower level microstructures were used for the calculation of thermal conductivity of higher level microstructures.

The outline of the article is as follows. In the next section, the multicomponent and multiphase phase-field model is described. The gas bubble evolution and predicted intergranular gas bubble structures is presented in the third section. In the fourth and fifth sections, the calculation of thermal conductivity in a polycrystalline material with heterogeneous thermal conductivity is described. The effect of intragranular and intergranular gas bubble structures on thermal conductivity is calculated. In the sixth section, the effective Kapitza resistance of the grain boundary with distributed intergranular gas bubbles is calculated. The effect of grain sizes on the thermal conductivity is assessed and compared with the experimental results. The seventh section discusses the effect of gas bubbles in recrystallized fuels on the thermal conductivity. Discussions of the calculations and conclusions are given in the final section.

\section{Description of multicomponent and multiphase phase-field model}

Gas bubble evolution in U-Mo metal fuel is a complicated process. The formation and growth of gas bubbles require continuous supplies of vacancies and gas atoms by diffusion while 
interstitials can hinder the nucleation and growth of gas bubbles through recombination or annihilation reactions. Since the free volume on the grain boundaries is large and the diffusivity of species is rapid, it is believed that gas bubbles prefer to nucleate and grow on grain boundaries. Intergranular gas bubbles usually grow much faster than intragranular gas bubbles. The eutectoid transition from gamma $\mathrm{U}$ to alpha plus gamma prime $\mathrm{U}$, the phase reversal due to neutron irradiation [23], and/or irradiation-induced recrystallization cause changes in grain size, hence, interfaces and gas bubble nucleation sites. Thermal and neutron-induced emission of gas atoms and vacancies from gas bubbles, voids, dislocations and grain boundaries may also affect the gas bubble evolution kinetics. Furthermore, internal pressure inside gas bubbles is high, especially in small bubbles. The elastic interaction between deformation associated with gas bubbles and deformation associated with fuel swelling might be important for gas bubble nucleation as well as growth kinetics. In this work, a generic multicomponent and multiphase phase-field model was used to describe gas bubble evolution. The model enables consideration of the diffusion of vacancies, gas atoms, Mo, and interstitials, the formation of gas bubbles, and phase transition from gamma $\mathrm{U}$ to alpha plus gamma prime $\mathrm{U}$. The concentrations of $\mathrm{U}$ vacancies, $\mathrm{Xe}$ gas atoms, $\mathrm{U}$ interstitials and Mo are described by molar-fraction fields, i.e., $\mathbf{c}=\left\{c_{1}(\mathbf{x}, t), c_{2}(\mathbf{x}, t), \cdots, c_{M}(\mathbf{x}, t)\right\}$ where $M$ is the total number of mobile species in the system. The spatial distribution of different phases such as gas bubbles, gamma, alpha and gamma prime phases as well as the orientation of matrix gamma phases are described by the order parameter fields, i.e., $\boldsymbol{\eta}=\left\{\eta_{1}(\mathbf{x}, t), \eta_{2}(\mathbf{x}, t), \cdots, \eta_{N}(\mathbf{x}, t)\right\}$ where $N$ is the total number of phases in the system (in a polycrystalline material, different orientated grains are denoted as different phases). In phase-field formulism the total free energy of the system, $F(\boldsymbol{\eta}, \mathbf{c})$, is described as a function of the order-parameter field $\boldsymbol{\eta}=\left\{\eta_{1}(\mathbf{x}, t), \eta_{2}(\mathbf{x}, t), \cdots, \eta_{N}(\mathbf{x}, t)\right\}$ and molar-fraction field $\mathbf{c}=\left\{c_{1}(\mathbf{x}, t), c_{2}(\mathbf{x}, t), \cdots, c_{M}(\mathbf{x}, t)\right\}[24,25]:$

$$
F(\boldsymbol{\eta}, \mathbf{c})=F_{s}(\boldsymbol{\eta})+F_{b}(\boldsymbol{\eta}, \mathbf{c})=\int_{V}\left[f_{s}(\boldsymbol{\eta})+f_{b}(\boldsymbol{\eta}, \mathbf{c})\right] d V
$$

where $\mathrm{V}$ is the volume of the system, $f_{b}$ is the chemical free energy density, and $f_{s}$ is the gradient energy density representing interfacial energy. The gradient energy density is written as

$$
f_{s}=m f_{0}(\boldsymbol{\eta})+\frac{\kappa}{2} \sum_{\rho=\alpha, \beta, \gamma}\left(\nabla \eta_{\rho}\right)^{2}
$$




$$
f_{0}(\boldsymbol{\eta})=\sum_{\rho}\left(\frac{\eta_{\rho}^{4}}{4}-\frac{\eta_{\rho}^{2}}{2}\right)+\sum_{\rho}\left(\sum_{\sigma \neq \rho} \frac{\gamma_{\rho, \sigma}\left(\psi_{\rho, \sigma}, \tilde{\boldsymbol{\mu}}\right)}{2} \eta_{\rho}^{2} \eta_{\sigma}^{2}\right)+\frac{1}{4}
$$

where $m, \kappa$ and $\gamma_{\rho, \sigma}\left(\psi_{\rho, \sigma}, \tilde{\boldsymbol{\mu}}\right)$ are model parameters determined by the properties of the interface between phase $\rho$ and $\sigma$. Parameters $m$ and $\kappa$ are determined by interfacial energy and interface thickness while $\gamma_{\rho, \sigma}\left(\psi_{\rho, \sigma}, \tilde{\boldsymbol{\mu}}\right)$ is determined by interfacial symmetry. Quantitative relationships between these model parameters and interfacial properties can be obtained by thin interface limit analysis $[25,26]$. The chemical free energy density is defined as

$$
f_{b}=\sum_{\alpha=1}^{N} h_{\alpha} f_{\alpha}\left(\mathbf{c}_{\alpha}\right)
$$

where $f_{\alpha}\left(\mathbf{c}_{\alpha}\right)$ is the chemical free energy of $\alpha$ phases. Different phases at point $\mathbf{x}$ are assumed to possess different concentrations, but have the same chemical potential $\partial f_{\alpha}\left(\mathbf{c}_{\alpha}\right) / \partial c_{\alpha i}=\partial f_{\beta}\left(\mathbf{c}_{\beta}\right) / \partial c_{\beta i},(\alpha, \beta=1, \ldots, N$, and $i=1,2, \ldots M)$ where $h_{\alpha}(\boldsymbol{\eta})$ is a shape function that is related to the volume fraction of $\alpha$ phase at point $\mathbf{x}$. In addition, in order to correctly describe the equilibrium properties at a triple point of three different phases, the shape function is defined as $h_{\alpha}(\boldsymbol{\eta})=\eta_{\alpha}^{2} / \sum_{\rho=1}^{N} \eta_{\rho}^{2}$. The microstructure evolution including gas bubbles and grains is controlled by the time dependent Cahn-Hilliard equations [27] and Allen-Cahn equations [28]:

$$
\begin{aligned}
& \frac{\partial c_{i}}{\partial t}=\nabla \cdot \sum_{j}\left(M_{i j} \nabla \frac{\delta F}{\delta c_{j}}\right)+\xi_{i}+\dot{g}_{i}+\dot{\gamma}_{i}+\dot{S}_{i}, \quad(i=1,2, \cdots, M) \\
& \frac{\partial \eta_{\rho}}{\partial t}=-L_{\rho} \frac{\delta F}{\delta \eta_{\rho}}+\xi_{\rho}, \rho=1,2, \cdots, N .
\end{aligned}
$$

where $M_{i j}$ and $L_{\rho}$ are mobility coefficients, and $\xi_{i}$ and $\xi_{\rho}$ are the thermal fluctuations. In equation (5a), $\dot{g}_{i}$ is the generation rate of defect $i, \dot{\gamma}_{i}$ is the reaction rate that is proportional to diffusivity and concentrations of defects, and $\dot{S}_{i}$ is the sink rate/strength. The $\dot{g}_{i}, \dot{\gamma}_{i}$ and $\dot{S}_{i}$ could use exactly the same expressions in the rate theory $[29,30]$.

By numerically solving these two groups of equations, we can obtain the spatial and temporal evolution of the phase field variables of $\mathbf{c}=\left\{c_{1}, c_{2}, \cdots, c_{M}\right\}$ and $\boldsymbol{\eta}=\left\{\eta_{1}, \eta_{2}, \cdots, \eta_{N}\right\}$, and then the microstructure. In this generic phase-field model, the chemical free energy density $f_{b}$ is a function of concentration and order parameter fields while the gradient energy density $f_{s}$ is described by order parameter fields shown in equations (2-3). The unknown parameters $m, \kappa$ 
and $\gamma_{\rho, \sigma}\left(\psi_{\rho, \sigma}, \tilde{\boldsymbol{\mu}}\right)$ are completely determined by the grain boundary and/or interface properties, and are independent of the concentration fields $\mathbf{c}=\left\{c_{1}(\mathbf{x}, t), c_{2}(\mathbf{x}, t), \cdots, c_{M}(\mathbf{x}, t)\right\}$ and chemical free energy $f_{b}$. One advantage of such a free energy formulation is that it is convenient in determining model parameters, especially for a multiphase and multicomponent system. The unknown model parameters can be quantitatively determined once the grain boundary properties including interface thickness, interfacial energy, and interface symmetry are known[25, 26]. Another advantage of such a model is that the chemical free energy $f_{\alpha}\left(\mathbf{c}_{\alpha}\right)$ of different phases, which can be obtaind from atomistic simulations and phase diagram calculations, can be directly used as shown in equation (4). Therefore, the model has potential application in simulating the concurrent evolution of multiphases in a system, for example, the evolution of gas bubble, gamma, alpha, gamma prime and grain growth in UMo metal fuels. In this work, we used the developed phase-field model to construct the intra- and inter-granular gas bubble structures in polycrystalline UMo, and then evaluated the effect of the initial polycrystalline structures and gas bubble structures on effective thermal conductivity. The density and mean radius of the gas bubbles observed at different fission densities was used to generate the initial distribution of gas bubbles in the simulation cell. The gas bubble structures were obtained by solving equation (5) to relax the interface of gas bubbles having equilibrium profiles. For example, the intergranular gas bubbles have a lens shape associated with the given interfacial energies. After that the obtained gas bubble structure was used for calculating the effective thermal conductivity. In all simulations the generation, reaction and sink of defects was not considered, which will be considered in simulating the gas bubble nucleation and growth kinetics in an irradiation environment in our future work.

\section{Construction of gas bubble structures}

From Figure 1 and TEM results [1], the gas bubble structure evolution is roughly separated into two different stages, i.e., before and after recrystallization. Before recrystallization, intragranular gas bubbles remain small (around 2 nanometers in diameter), but gas bubble density increases as a result of continuous high-energy particle radiation-induced dissolution of larger gas bubbles and the continuous nucleation of gas bubbles. However, the intergranular gas bubbles increase in size with increasing fission density. During this stage, the gas bubble structures are described by intragranular and intergranular gas bubbles. Once the recrystallization takes place, the formation of small grains leads to the increase of grain boundaries that increases the nucleation sites of intergranular gas bubbles as well as their coarsening kinetics. The intergranular gas bubbles structure inside the recrystallization zone looks like an uniform distribution of gas bubbles as shown in Figure 1b. The recrystallization zone expands with increasing fission density. Eventually all coarse grains become fine grains, and the intergranular gas bubbles distribute uniformly as shown in Figure 1c. The intergranular gas bubbles inside the recrystallization zone 
can be described by a mean diameter and density like the intragranular gas bubbles. Table 1 lists the mean radius and density at different fission densities. The measurements were estimated from data reported in reference [31, 32] and optical microscopy images [33]. The intergranular gas bubble density is measured in per unit area of the grain boundary. The gas bubble radius is given as the average value.

Considering that intragranular gas bubbles are two or three orders of magnitude smaller than that of intergranular gas bubbles, it is not practical to construct both inter- and intra-gas bubbles in one simulation cell that requires a very fine grid size to capture the nanometer sized intragranular gas bubbles, and dramatically increases the computation cost. Therefore, our stratagem is that we will use the phase-field model to construct different gas bubble structures including intragranular gas bubbles in single crystals, intergranular gas bubbles in polycrystals, and gas bubbles inside recrystallization zones in polycrystals, respectively, and then evalyuate their individual and coupling effect on thermal conductivity by numerical and semi-emperical formula. The purpose of using phase-field models to construct the gas bubble structures is to obtain complicated three dimensional microstructures including shape and size distributions observed in the experiments. To do so, the concentrations inside gas bubbles and matrix were set to be equilibrium concentrations in the simulations. As a concequence, the gas bubble evolution controlled by equation (5) will only change the morphology (the shape of the gas bubble), which is determined by the interfacial energy and/or grain boundary energy, but keep the size and the volume fraction before the gas bubble coarsening takes place.

For intragranular gas bubbles, we randomly introduce spherical gas bubbles with $2 \mathrm{~nm}$ in diameter into a single crystal with a $256 d x \times 64 d y \times 64 d z$ simulation cell. The grid size $d x=d y=d z=0.3 \mathrm{~nm}$ is used. Different gas bubble densities from $3 \times 10^{24} \mathrm{bubbles} / \mathrm{m}^{3}$ to $1 . \times 10^{25}$ bubbles $/ \mathrm{m}^{3}$ are considered. In order to construct the intergranular gas bubbles in a polycrystal, three steps were taken. At the first step, the phase-field model of grain growth, which is described by equation (5) by eliminating concentration fields, was used to generate a polycrystalline structure. At the second step, the intergranular gas bubbles were introduced into the polycrystalline structure obtained at the first step. And at the third step, the phase-field model of gas bubble evolution, which is described by equation (5) with order parameter and concentration fields, was used to relax the intergranular gas bubbles to have equilibrium wetting angles which is determined by the given interfacial energies, and output the intergranular gas bubble structure which will be used to calculate the thermal conductivity. In the simulations, a $200 d x \times 200 d y \times 200 d z$ simulation cell with $d x=d y=d z=30 n m$ was used. Three different size distributions of intergranular gas bubbles are presented in Figure 2 [31] and different densities from $5 \times 10^{11}$ to $3 \times 10^{12}$ bubbles $/ \mathrm{m}^{2}$ are used as the input. Since the grain boundary energy of U10 Mo alloys and interfacial energy between gas bubble and U-10Mo matrix is not available in literature, the grain boundary energy of $0.5 \mathrm{~J} / \mathrm{m}^{2}$ [31] and interfacial energy between gas bubble and matrix of $0.36 \mathrm{~J} / \mathrm{m}^{2}$ were assumed. For simplicity, it is also assumed that the interface and 
grain boundary are symmetrical, so $\gamma_{\rho, \sigma}\left(\psi_{\rho, \sigma}, \tilde{\boldsymbol{\mu}}\right)$ is set to be 1.5 [26]. For the relaxation of gas bubble structures, the interface properties which determine the morphology of gas bubbles are important while chemical free energy is not important which affects the driving force of gas bubble evolution, i.e., the kinetics. Therefore, two paraballic functions $f_{\alpha}\left(\mathbf{c}_{\alpha}\right)$, which have the common tangent at the equilibrium concentrations, are used to describe the free energies of gas bubble and matrix phases. For the construction of intergranular gas bubble structures, 24,000 simulation steps were used that ensures that an isolated gas bubble has an equilibrium wetting angle expected from the interfacial energies while 2,000 simulation steps were used to construct the intragranular gas bubble structures. Figure 2 shows the evolution of intergranular gas bubbles with an initial density $1.4 \times 10^{12}$ bubbles $/ \mathrm{m}^{2}$ and an average radius $80 \mathrm{~nm}$ from sphere to lens-like shapes. Some gas bubbles are observed to coalesce and connect to form channels. In order to describe the intergranular gas bubble structure, a structural variable $f_{\text {cov }}$ is defined to measure the interfacial coverage by the gas bubbles that is the ratio of the area occupied by gas bubble to the total grain boundary area in the polycrystal. The intergranular gas bubble structures with different densities created by phase-field simulations are shown in Figure 3.

The recrystallization zone forms first on grain boundaries and then propagates inside grains. In order to mimic the recrystallization zone evolution and construct the gas bubble structure inside the recrystallization zone in a polycrystal, the evolution of a vacancy concentration field in a polycrystal was simulated and the evolving vacancy concentration field was used to define the time evolution of the recrystallization zone. To do so, an initially constant vacancy concentration was set up in the polycrystal, after that the diffusion equation (5a) was solved with the assumption that grain boundaries were the sink of vacancies, i.e., the vacancy concentration at grain boundaries maintains thermal equilibrium concentration by removing the extra vacancy. Thus vacancy diffusion will generate a spatial distribution of vacancy concentration in the polycrystal. Based on vacancy concentration contours, different recrystallization zones can be defined. In the simulations, we consider a polycrystal with dilute vacancies. The phase-field model described above has $N$ order parameter fields that describe $N$ different orientated grains and one vacancy field. Each grain has the same chemical free energy $f(c)$ that has the format of ideal solution free energy. The equilibrium vacancy concentration is set to be 1.e- 6 while the initial constant vacancy concentration is set to be 0.001 . The region where vacancy concentration is less than 0.0001 is defined as the recrystallization zone. The average gas bubble sizes and volume fraction inside the recrystallization zone listed in Table 1 were used to calculate the gas bubble density. Then, the gas bubble structure is obtained by introducing the gas bubbles into recrystallization zones and relaxing the gas bubble with equation (5). Figure 4 shows the created gas bubbles within different recrystallization zones with the average gas bubble size and density observed experimentally [33]. It should be pointed out that the proposed method only provides one way to define recrystallization zones in a three dimensional polycrystal. In this work we didn't simulate the recrystallization process. To develop a phase-field model of recrystallization needs the fundamental understanding of the recrystallization mechanisms that are not well 
known. Therefore, future effort in understanding recrystallization mechanisms and predicting recrystallization zone evolution kinetics are required in order to fuel performance.

\section{Calculation of Effective Thermal Conductivity}

The thermal conductivity of Xe gas bubbles and U-10Mo have been experimentally evaluated. Figure 5 shows the thermal conductivity as a function of temperature reproduced from experimental data $[2,6]$. The unirradiated thermal conductivity of U-Mo alloys is about two orders of magnitude larger than that of Xe gas bubbles. In addition, the thermal conductivity on grain boundaries and interfaces between gas bubbles and matrix will also be much smaller than that of U-Mo alloys. For simplicity, the inhomogeneous thermal conductivity in a polycrystal material with gas bubbles is described as [21]

$$
\kappa(\boldsymbol{\eta})=\kappa_{G}\left(\sum_{i=2}^{p} \eta_{i}^{2}\right)^{n}+\kappa_{g a s} \eta_{1}^{2}
$$

where $\kappa_{G}$ and $\kappa_{\text {gas }}$ are the thermal conductivity of single crystal U-Mo and gas phase, respectively. The variables $\eta_{1}$ and $\eta_{i}(i=2, \ldots N)$ are the order parameters of gas bubble and grains with different orientations, respectively. The order parameters $\eta_{1}$ and $\eta_{i}$ are read from the output of phase-field modeling of gas bubble structures in the previous section. The exponent $n$ is an adjustable parameter to fit the thermal conductivity at the grain boundary and/or interface between gas bubble and matrix.

In order to calculate the effect of gas bubbles on thermal conductivity, heat transportation in a polycrystal material as shown in Figure 6 was considered. The temperatures at two ends in the $x$ axis of the simulation cell were set to $T_{1}$ and $T_{2},\left(T_{1}>T_{2}\right)$, respectively. The middle part is the polycrystal material with distributed gas bubbles that are obtained from phase-field simulations in the previous section. Solving the following heat transport equation, the steady state solution of temperature field $\mathrm{T}$ is obtained

$$
\frac{\partial T}{\partial t}=\nabla \cdot(\kappa(\boldsymbol{\eta}) \nabla T)
$$

where $\kappa\left(\boldsymbol{\eta}_{i}\right)$ is the local thermal conductivity calculated by equation (6). The effective thermal conductivity $\kappa^{e f f}$ of the polycrystal material with distributed gas bubbles can be calculated by

$$
\kappa^{e f f}=J_{Q} /(d T / d x)
$$


where $J_{Q}$ is the average heat flux and $d T / d x$ is the average temperature gradient along the $\mathrm{x}$ axis. The average heat flux is calculated as $J_{Q}=\left.\iint_{S} \kappa(\boldsymbol{\eta}) \nabla T d s\right|_{x=x_{0}} / S_{0}$, where $s$ is the cross section of the simulation cell at $x=x_{0}$, and $S_{0}$ is the area of the cross section. This method has been used to study the effect of gas bubbles and voids on thermal conductivity in two dimensions $[18,20,21]$.

In this work we assess thermal conductivity at temperature $T=423 \mathrm{~K}$ that is close to the metal fuel operation temperature. From Figure 5, the thermal conductivity measured in polycrystalline $\mathrm{U}-10 \mathrm{Mo}$ samples is $\kappa=15.0 \mathrm{~W} / \mathrm{mK}$. The thermal conductivity of $\mathrm{Xe}$ is about $\kappa_{\text {gas }}=0.008 \mathrm{~W} / \mathrm{mK}$. Unfortunately, experimentally measured thermal conductivity of single crystal U-10Mo $\kappa_{G}$ in literature as well as the grain boundary thermal conductivity was not found. The two unknown parameters $\left(\kappa_{G}\right.$ and $\left.n\right)$ in equation (6) are determined first before the effect of gas bubbles on thermal conductivity is calculated. For simplicity, the exponent $n$ is set to be six in this work that makes the minimum thermal conductivity at interface between gas bubble and matrix less than that of Xe phase. The grain size dependence of thermal conductivity of a polycrystalline material can be expressed as $[34,35]$

$$
\kappa^{e f f}=\frac{\kappa_{G}}{1+\frac{\kappa_{G} R_{G B}}{d}}=\frac{\kappa_{G}}{1+\frac{L_{k}}{d}}
$$

where $d$ is the average grain size, $R_{G B}$ is the Kapitza resistance of grain boundary, and $L_{k}$ is the Kapitza length of the grain boundary that is calculated by

$$
L_{k}=\kappa_{G} R_{G B}=\frac{\kappa_{G} \Delta T}{J_{G B}}
$$

where $J_{G B}$ is the heat flux $\left(W / \mathrm{m}^{2}\right)$ and $\Delta T$ is the temperature drop cross the grain boundary. The expression has been employed to determine the average interface Kapitza resistance $R_{G B}$ for polycrystalline materials with their measured conductivity.

To assess $\kappa_{G}$ a bi-crystal was used to calculate the Kapitza length $L_{k}$. In the simulations, the following model parameters were used: simulation cell $1024 d x \times 1 d x \times 1 d x, T_{1}=500 \mathrm{~K}$, $T_{2}=300 \mathrm{~K}$, and $d x=30 \mathrm{~nm}$. For a given $\kappa_{G}=17.5,18.0$, and $18.5 \mathrm{~W} / \mathrm{mK}$, the heat flux, $J_{G B}$, and the temperature drop cross the grain boundary, $\Delta T$, can be obtained from the simulation in the bi-crystal. The Kapitza length of the grain boundary was calculated with equation (10), and the effective thermal conductivity of polycrystalline materials was assessed by equation (9). 
Figure 7 shows the grain size dependence of effective thermal conductivity for different thermal conductivity $\kappa_{G}$. From the results we find that if $\kappa_{G}=18.0 \mathrm{~W} / \mathrm{mK}$ is used, the effective thermal conductivity of the polycrystalline material fits well with the experimentally measured thermal conductivity $\kappa=15.0 \mathrm{~W} / \mathrm{mK}$. Thus, in the following calculations $\kappa_{G}=18.0 \mathrm{~W} / \mathrm{mK}$ was used.

\section{Effect of gas bubbles on thermal conductivity}

As a result of the significant size difference between intra- and inter- granular gas bubbles, the calculation of the effective thermal conductivity of a polycrystalline material with intra- and inter-granular gas bubbles was separated into two steps. In the first step the thermal conductivity of a single crystal with nanometer sized intragranular gas bubbles was calculated. In the second step the thermal conductivity of a polycrystalline material with intra- and inter-granular gas bubbles was calculated, but replacing the intragranular gas bubbles with the effective thermal conductivity determined in the first step.

\subsection{Intragranular gas bubbles}

Two gas bubble structures are constructed in single crystals. One assumes uniformly distributed nanometer sized gas bubbles, and the other assumes a gas bubble layer with a thickness of $10 \mathrm{~nm}$. A simulation cell of $256 d x \times 64 d y \times 64 d z$ and $d x=d y=d z=0.3 \mathrm{~nm}$ was used in the simulations. The phase-field model was used to create the gas bubble structure that consists of gas bubbles with 2 nanometers in diameter and different densities. Inserting the single crystal with gas bubble structures into the simulation cell of thermal conductivity calculation shown in Figure 6, assigning inhomogeneous thermal conductivity according to equation (6), the thermal conductivity is then calculated by solving equations (7-8). Figure 8 plots the effect of gas bubble density on the effective thermal conductivity. It is interesting to find that gas bubbles within a layer with $10 \mathrm{~nm}$ thickness do not significantly affect the thermal conductivity when the simulated gas bubble density varies from densities of $3 \times 10^{24} \sim 1 \times 10^{25}$ bubbles $/ \mathrm{m}^{3}$. However, the effective thermal conductivity linearly decreases with increasing gas bubble density for the uniformly distributed gas bubble structures.

Some empirical models and formula have been developed to evaluate the effect of voids or gas bubbles on theremal conductivity. Kampf and Peddicord [8-11] developed analytical porosity correlations of thermal conductivity for structures with distributed cubic and spherical pores. The effective thermal conductivity can be calculated by $K=K_{0} \exp (-2.14 p)$, where $K_{0}$ is the thermal conductivity of $100 \%$ dense materials and $p$ is the porosity. Bauer [12] proposed a empirical relationship between effective thermal conductivity and gas bubble volume fraction

$K=K_{0}(1-p)^{2.58}$. These empirical models are applicable to intragranular gas bubbles that have very small thermal conductivity and uniform distribution. A comparison of the results from 
current work and calculated by the empirical formula is presented in Figure 8. It is found that our results for distributed intragranular gas bubbles are in acceptable agreement with the prediction by the Bauer's model. The Peddicord's model gives a overestimation for large void volume fraction compared to our results and Bauer's model. The comparison demonstrates that the parameters used in our simulations such as the interface thermal conductivity model are reasonable.

\subsection{Intergranular gas bubbles}

In section 3 the intergranular gas bubble structures were obtained by the phase-field simulations. Using the gas bubble structures, the thermal conductivity is assigned according to equation (6), but $k_{G}$ is now replaced by the effective thermal conductivity of a single crystal with intragranular gas bubbles that was calculated in section 5.1. Since experimentally measured intergranular gas bubble distribution and density were used as the initial input to obtain the gas bubble structure, a structural variable $f_{\text {cov }}$, named as grain boundary coverage, was introduced to describe the intergranular gas bubble structure. The grain boundary coverage is defined as

$$
f_{\mathrm{cov}}=\frac{S_{G B}^{g a s}}{S_{G B}}
$$

where $S_{G B}^{\text {gas }}$ are the area covered by the intergranular gas bubbles, and $S_{G B}$ is the total grain boundary area. Figure 9 shows the effect of inter- and intra-granular gas bubbles on the effective thermal conductivity. The three dimensional microstructures correspond to the grain boundary coverages, respectively. The value of grain boundary coverage increases with the increase of fission density, while the value of effective thermal conductivity $k_{G}$ decreases. The effective thermal conductivity $k_{G}=13,15$, and $17 \mathrm{~W} / \mathrm{mK}$ corresponds to intragranular gas bubble density $2 \times 10^{24}, 6 \times 10^{24}$, and $1 \times 10^{25}$ bubbles $/ \mathrm{m}^{3}$, respectively. The thermal conductivity of the polycrystalline material without intergranular gas bubbles is $13 \mathrm{~W} / \mathrm{mK}$ which is smaller than $15 \mathrm{~W} / \mathrm{mK}$ measured in the experiments. The reason is that in the simulation the grain size is about 2 micrometers while the grain size in the experimental samples are around 20 micrometers. In addition, $k_{G}=17 \mathrm{~W} / \mathrm{mK}$ is used in the simulation implying there was an intragranular gas bubble density of $2 \times 10^{24}$ bubbles $/ \mathrm{m}^{3}$. It is found that the thermal conductivity decreases with the increase of grain boundary gas bubble coverage and intragranular gas bubble density (i.e., increasing fission density). The thermal conductivity with $30 \%$ interface coverage and intragranular gas bubbles (diameter $2 \mathrm{~nm}$ and density $1 \times 10^{25}$ bubbles $/ \mathrm{m}^{3}$ ) is $8.1 \mathrm{~W} / \mathrm{mK}$. The reduction of the thermal conductivity compared to the polycrystalline material is about $38 \%$.

\subsection{Effect of grain morphology on thermal conductivity}


Rolling, that is a key process during the metallic fuel foil fabrication, elongates grains along the rolling direction. In order to assess the effect of grain morphology on the thermal conductivity, grains in $x$-direction were elongated and those in the $y$-direction were compressed, and remain the same in $z$-direction. We consider a case that the original simulation cell $200 d x \times 200 d x \times 200 d x$ becomes $400 d x \times 100 d x \times 200 d x$ after the deformation. First the equiaxed polycrystal was deformed to have elongated grains, then the gas bubbles were introduced into the polycrystal. The same methods described in previous sections are used to construct the intra- and inter- granular gas bubbles, and calculate the effective thermal conductivity. The thermal conductivity along the elongated grain direction and the compressed grain direction were calculated as a function of different gas bubble grain boundary coverages, respectively. The results are presented in Figure 10. In the simulations, $k_{G}=18 \mathrm{~W} / \mathrm{mK}$ was used, i.e., there are no intragranular gas bubbles. The red square dotted line shows the thermal conductivity as a function of grain boundary coverage along the elongated direction shown by the red arrow in the inserted microstructure. The green triangle dotted line shows the thermal conductivity as a function of grain boundary coverage along the compressed direction shown by the green arrow in the inserted microstructure. The thermal conductivity of equiaxed grains was also plotted as the blue line for comparison. It is interesting to find that the thermal conductivity along both elongated and compressed directions are smaller than that of polycrystalline materials with equiaxed grains. The thermal conductivity along the compressed direction has $67 \%$ reduction. The results demonstrate that the grain elongation formed during rolling process may cause the strong anisotropy of thermal conductivity.

\section{Effect of grain size on thermal conductivity}

In order to capture the morphology of intergranular gas bubbles, a small grid size that limits the physical domain size has to be used for the effective thermal conductivity calculations. As a result, the grain size in the polycrystalline materials in the current simulations is around $2 \mu m$. However, in U-Mo fuels, the grain size is usually tens to hundreds of micrometers. To assess the effect of grain size on the thermal conductivity, the effective thermal conductivity of grain boundaries with distributed intergranular gas bubbles was calculated. To do so, we consider a bicrystal in a simulation cell $256 d x \times 64 d x \times 64 d x$, where $d x=30 \mathrm{~nm}$ is used. On the grain boundaries we introduce intergranular gas bubbles with different densities, obtain the equilibrium gas bubble structure, and calculate the thermal conductivity as described in section 5.2. Figure 11 shows the effect of gas bubble grain boundary coverage and grain thermal conductivity on the effective thermal conductivity of grain boundaries. The average temperature $T(x)$ at the cross section $x$ is calculated. Figure 12 plots the average temperature drop across the grain boundary, which is approximately $4 \mathrm{~K}$. The temperature drop increases with increasing gas bubble grain boundary coverage that is shown in Figure 13a. Using the temperature drop $\Delta T$, and heat flux $J_{G B}$, the effective Kapitza resistance at the grain boundary was calculated by 
$R_{G B}=\frac{\Delta T}{J_{G B}}$. The results are presented in Figure 13b. The grain size dependence of thermal conductivity can be calculated by equation (10) where $\kappa_{G}$ is the effective thermal conductivity of a single crystal with distributed nanometer sized gas bubbles calculated in section 5.1, and $d$ is the grain diameter.

Figure 14a-d shows the effect of the gas bubble grain boundary coverage $f_{\text {cov }}$, the thermal conductivity $\kappa_{G}$ of a single crystal that is associated with the density of intragranular gas bubbles, and grain size. It is found that the effective thermal conductivity reaches constant values with increased grain size. The vertical dashed lines mark the position where the average grain size is about $2 \mu m$ that was used in three-dimensional simulations presented thus far. Comparing Figure 9 and Figure 14, it is found that the results assessed by three-dimensional simulations are in good agreement with that calculated by the empirical formula (equation (9)). Figure 15 summarizes the thermal conductivity of polycrystalline materials with coarse grains. The dashed line schematically directs the changes of $f_{\text {cov }}$ and $\kappa_{G}$ during the operation process ( $f_{\text {cov }}$ increases while $\kappa_{G}$ decreases). The symbol "star" presents the experimental data for three different burnup stages [33], i.e., before recrystallization, partial recrystallization and full recrystallization. In previous calculations, only the intra- and inter-granular gas bubbles were considered. This implies that the results are only applied to the materials before recrystallization occurs. In next section we will consider the thermal conductivity calculation of the materials after recrystallization. We can see that the first experimental point before recrystallization acceptably falls into the region predicted by the simulations.

\section{Effect of recrystallization on thermal conductivity}

Gas bubble structures within different recrystallization zones were constructed in section 3 to represent the period following recrystallization. With these gas bubble structures, the thermal conductivity was assessed assuming two extreme cases. In one case the effect of grain boundaries in the recrystallization zone on thermal conductivity was taken into account, and in the other case the effect of grain boundaries in the recrystallization zone on thermal conductivity was ignored. The first case corresponds to the microstructure with dense grain boundaries and gas bubbles while the second case corresponds to the microstructure with only dense gas bubbles. These two cases should provide the lower and upper bounds of thermal conductivity, respectively. The results are plotted in Figure 16 where the red triangle dots and blue square dots present the upper and lower bounds of thermal conductivity, respectively. The scattered simulation points are due to different runs for a system with the same gas bubble volume fraction but different spatial and size distributions of gas bubbles. The "star" symbol again represents the 
experimental data [33]. It can be seen that the experimental data follow in the region between the predicted upper and lower bounds of effective thermal conductivity. The predicted lower bound of thermal conductivity implies that recrystallization, which generates fine grains and importantly increases the grain boundary fraction in the system, may result in a large thermal conductivity reduction.

\section{Discussions}

In this work we presented a method to assess thermal conductivity of a polycrystalline material with inter- and intra- granular gas bubbles. We used a phase-field model to construct three dimensional polycrystals and different gas bubble structures. The effect of gas bubble structures on effective thermal conductivity of single crystal, grain boundary and polycrystalline materials was numerically investigated. Combining the calculated effective thermal conductivity and empirical formula, the relative effect of grain sizes, grain morphology and recrystallization stages on thermal conductivity were assessed. The upper and lower bounds of thermal conductivity were predicted by the simulations. The most interesting results are that 1) simulated effect of intragranular gas bubbles on thermal conductivity is in acceptable agreement with that calculated from Bauer's model; 2) the elongated grains formed during rolling may result in thermal conductivity degradation and strong anisotropy of thermal conductivity, and 3) the recrystallization may cause about $60 \%$ thermal conductivity reduction. The comparison between predicted and valid experimental results demonstrates that the developed method can reasonably predict the effect of complicated microstructures produced during fabrication on thermal conductivity. However, in order to more quantitatively predict the thermal conductivity degradation during fuel operations, it is essential 1) to assess the effective thermal conductivity of interface and grain boundary by benchmarking the results from atomistic simulations, mesoscale phase-field simulation and experiments, and 2) to predict the gas bubble evolution kinetics in irradiated fuels for the input of effective thermal conductivity calculation.

\section{Acknowledgements}

The work described in this article was performed by Pacific Northwest National Laboratory, which is operated by Battelle for the United States Department of Energy under Contract DEAC05-76RL01830. This study was supported by the U.S. Department of Energy, National Nuclear Security Administration, Office of Material Management and Minimization Reactor Conversion Program.

\section{References}

[1] S. Neogy, M. T. Saify, S. K. Jha, D. Srivastava, M. M. Hussain, G. K. Dey, and R. P. Singh, Journal of Nuclear Materials 422 (2012) 77. 
[2] D. E. Burkes, C. A. Papesch, A. P. Maddison, T. Hartmann, and F. J. Rice, Journal of Nuclear Materials 403 (2010) 160.

[3] S. Van den Berghe, W. Van Renterghem, and A. Leenaers, Journal of Nuclear Materials 375 (2008) 340.

[4] Y. S. Kim and G. L. Hofman, Journal of Nuclear Materials 419 (2011) 291.

[5] D. E. Burkes, G.S. Mickum, and D. M. Wachs, INL/EXT-10-19373 (2010)

[6] V. K. Saxena and S. C. Saxena, Journal of Chemical Physics 51 (1969) 3361.

[7] J. C. Maxwell, ed., Treatise on Electricity and Magnetism, Vol. 1, Oxford University Press, Oxford, 1873/1904.

[8] H. Kampf and G. Karsten, Nuclear Applications and Technology 9 (1970) 288.

[9] K. L. Peddicord, M. E. Cunningham, and A. Tripathi, Transactions of the American Nuclear Society 28 (1978) 548.

[10] K. L. Peddicord and M. Ades, Transactions of the American Nuclear Society 28 (1978) 552.

[11] T. H. Bauer and J. W. Holland, Nuclear Technology 110 (1995) 407.

[12] T. H. Bauer, International Journal of Heat and Mass Transfer 36 (1993) 4181.

[13] D. Yun, A. M. Yacout, M. Stan, T. H. Bauer, and A. E. Wright, Journal of Nuclear Materials 448 (2014) 129.

[14] P. C. Millett, M. R. Tonks, K. Chockalingam, Y. F. Zhang, and S. B. Biner, Journal of Nuclear Materials 439 (2013) 117.

[15] M. C. Teague, B. S. Fromm, M. R. Tonks, and D. P. Field, Jom 66 (2014) 2569.

[16] A. Artemev, Y. M. Jin, and A. G. Khachaturyan, Philosophical Magazine a-Physics of Condensed Matter Structure Defects and Mechanical Properties 82 (2002) 1249.

[17] L. Q. Chen, Annual Review of Materials Research 32 (2002) 113.

[18] S. Y. Hu, C. H. Henager, H. L. Heinisch, M. Stan, M. I. Baskes, and S. M. Valone, Journal of Nuclear Materials 392 (2009) 292.

[19] S. Y. Hu, C. H. Henager, K. J. Geelhood, and W. G. Luscher, Am. Nucl. Soc. Trans. 98 (2008) 1130.

[20] P. C. Millett and M. Tonks, Journal of Nuclear Materials 413 (2011) 136.

[21] P. C. Millett, D. Wolf, T. Desai, S. Rokkam, and A. El-Azab, Journal of Applied Physics 104 (2008)

[22] K. Chockalingam, P. C. Millett, and M. R. Tonks, Journal of Nuclear Materials 430 (2012) 166.

[23] M. L. Bleiberg, Journal of Nuclear Materials 1 (1959) 182.

[24] J. Heulens, B. Blanpain, and N. Moelans, Acta Materialia 59 (2011) 3946.

[25] N. Moelans, B. Blanpain, and P. Wollants, Physical Review Letters 101 (2008)

[26] N. Moelans, B. Blanpain, and P. Wollants, Physical Review B 78 (2008)

[27] J. W. Cahn, Acta Metallurgica 9 (1961) 795.

[28] J. W. Cahn and S. M. Allen, J. de Physique 38 (1977) C7.

[29] C. H. Woo and B. N. Singh, Philosophical Magazine a-Physics of Condensed Matter Structure Defects and Mechanical Properties 65 (1992) 889.

[30] S. I. Golubov, A. V. Barashev, and R. E. Stoller, Comprehensive Nuclear Materials, 2012.

[31] J. Rest, Journal of Nuclear Materials 407 (2010) 55.

[32] J. Rest, G. L. Hofman, and Y. S. Kim, Journal of Nuclear Materials 385 (2009) 563.

[33] D. E. Burkes, A. M. Casella, E. C. Buck, A. J. Casella, M. K. Edwards, P. J. MacFarlan, K. N. Pool, B. D. Slonecker, F. N. Smith, F. H. Steen, and R. E. Thornhill, Pacific Northwest National Laboratory, Report PNNL-22981 (2013)

[34] H. S. Yang, G. R. Bai, L. J. Thompson, and J. A. Eastman, Acta Materialia 50 (2002) 2309.

[35] C. W. Nan and R. Birringer, Physical Review B 57 (1998) 8264.

[36] J. Rest, Y. S. Kim, G. L. Hofman, M. K. Meyer, and S. L. Hayes, ANL-09/31 (2009) http://www.osti.gov/bridge. 


\section{Figure Captions:}

Figure 1. SEM micrographs showing the gas bubble evolution with burnup in U-10wt\% Mo[4]. The darkest spots are fission gas bubbles. (a) fission density (FD) $=2.4 \times 10^{27} \mathrm{f} / \mathrm{m}^{3}$; (b) (FD)= $=2.9 \times 10^{27} \mathrm{f} / \mathrm{m}^{3} ;(\mathrm{c})(\mathrm{FD})==5.5 \times 10^{27} \mathrm{f} / \mathrm{m}^{3}$.

Figure 2. Temporal evolution of intergranular gas bubbles with an initial density $1.4 \times 10^{12}$ bubbles $/ \mathrm{m}^{2}$ and an average radius of $80 \mathrm{~nm}$. (a) three dimensional (3D) views of gas bubbles and grains after some grains were artificially hidden in plotting in order to clearly show the intergranular gas bubble distribution; (b) 3D views of gas bubbles only; and (c) 2D views of gas bubbles and grains at the cross section A shown in (a).

Figure 3. Constructed intergranular gas bubble structures with different densities, case 1: $5 \times 10^{11}$ bubbles $/ \mathrm{m}^{2}$, case $2: 1 \times 10^{12}$ bubbles $/ \mathrm{m}^{2}$ and case $3: 1.4 \times 10^{12}$ bubbles $/ \mathrm{m}^{2}$ by phase-field simulations. (a) $3 \mathrm{D}$ views of gas bubbles and grains after some grains were artificially hidden in plotting; (b) 2D views of gas bubbles and grains at the cross section A shown in (a).

Figure 4. Gas bubble structures in recrystallization zones with increasing time created by phasefield simulations. (a) 3D views of gas bubbles and grains after some grains were artificially hidden in plotting; (b) 2D views of gas bubbles and grains at the cross section A shown in (a).

Figure 5. (a) Thermal conductivity of unirradiated U-10Mo alloys versus temperature reproduced from reference [2, 36] and (b) thermal conductivities of fission gases versus temperature reproduced from reference[6].

Figure 6. Simulation cell of effective thermal conductivity calculation.

Figure 7. The grain size dependence of polycrystalline effective thermal conductivity for a given single crystal's thermal conductivity $\kappa_{G}$ and model parameters used.

Figure 8. The effect of intragranular gas bubble density on the effective thermal conductivity in a single crystal. The symbol lines represent the results from the Peddicord's model[9] and Bauer's model[11].

Figure 9. Calculated effective thermal conductivity of polycrystalline materials as a function of interface coverage of intergranular gas bubbles and effective thermal conductivity $K_{G}$ of grains with nano-sized intragranular gas bubbles.

Figure 10. Effect of grain morphology and intergranular gas bubbles on thermal conductivity. 
Figure 11. Effect of gas bubble interface coverage and effective thermal conductivity of grains $\left(\mathrm{K}_{\mathrm{G}}\right)$ on effective thermal conductivity of grain boundaries.

Figure 12. (a) Average temperature fields along $x$-direction of the simulation cell in Figure 6 for different interface coverage $f_{\text {cov }}$ and (b) temperature drop $\Delta T$ across the grain boundary in the region marked by the dashed lines in (a).

Figure 13. Effect of interface coverage $f_{\text {cov }}$ and effective thermal conductivity of grains $\left(\mathrm{K}_{\mathrm{G}}\right)$ on (a) temperature drop across the grain boundary and (b) Effective Kapitza Resistance at grain boundary $\left(\mathrm{R}_{\mathrm{GB}}\right)$.

Figure 14. Effect of average grain size, effective thermal conductivity of grains $\left(\mathrm{K}_{\mathrm{G}}\right)$ and different interface coverages $f_{\text {cov }}$ on thermal conductivity (a) $f_{\text {cov }}=0.0$; (b) $f_{\text {cov }}=0.21$; (c) $\mathrm{f}_{\text {cov }}=0.40 ;(\mathrm{d}) \mathrm{f}_{\text {cov }}=0.71$.

Figure 15. Predicted effective thermal conductivity as a function of gas bubble interface coverage and intragranular gas bubble density that is related to $\mathrm{K}_{\mathrm{G}}$. The experimental data is presented by symbol "star".

Figure 16. Predicted upper and lower bounds of thermal conductivity as a function of volume fraction. 


\section{Tables:}

Table 1 Observed gas bubble structures under different fission density in U-10Mo.

\begin{tabular}{|c|c|c|c|c|c|c|}
\hline \multirow[t]{2}{*}{$\begin{array}{l}\text { Fission density } \\
\left(f / m^{3}\right)\end{array}$} & \multicolumn{2}{|c|}{$\begin{array}{l}\text { Intragranular gas } \\
\text { bubble }\end{array}$} & \multicolumn{3}{|c|}{ Intergranular gas bubble } & \multirow[t]{2}{*}{$\begin{array}{l}\text { Recrystall } \\
\text {-ization }\end{array}$} \\
\hline & $\begin{array}{l}\text { Radius } \\
(\mathrm{nm})\end{array}$ & $\begin{array}{l}\text { Density } \\
\left(\text { bubbles } / \mathrm{m}^{3}\right)\end{array}$ & $\begin{array}{l}\text { Radius } \\
(\mathrm{nm})\end{array}$ & $\begin{array}{l}\text { Density } \\
\left(\text { bubbles } / \mathrm{m}^{2}\right)\end{array}$ & $\begin{array}{l}\text { Volume } \\
\text { fraction }\end{array}$ & \\
\hline $2.50 \times 10^{27}$ & 1 & $3 \times 10^{24}$ & 81 & $1.4 \times 10^{12}$ & & No \\
\hline $3.71 \times 10^{27}$ & 1.55 & $2.72 \times 10^{24}$ & 118 & $2.86 \times 10^{11}$ & $9.9 \%$ & Yes \\
\hline $4.08 \times 10^{27}$ & 1.57 & $2.68 \times 10^{24}$ & 148 & $3.39 \times 10^{11}$ & $22.9 \%$ & Yes \\
\hline
\end{tabular}




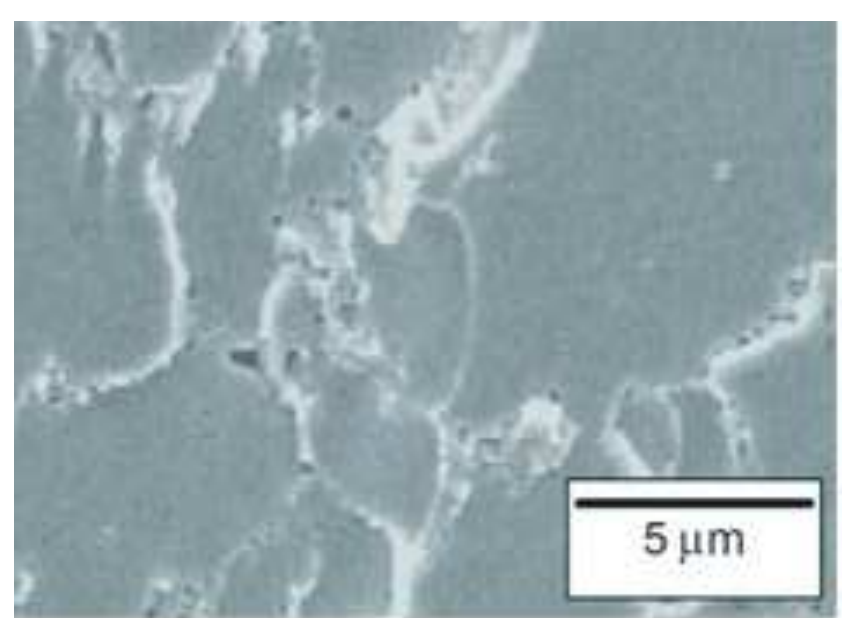

(a)

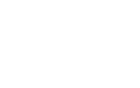

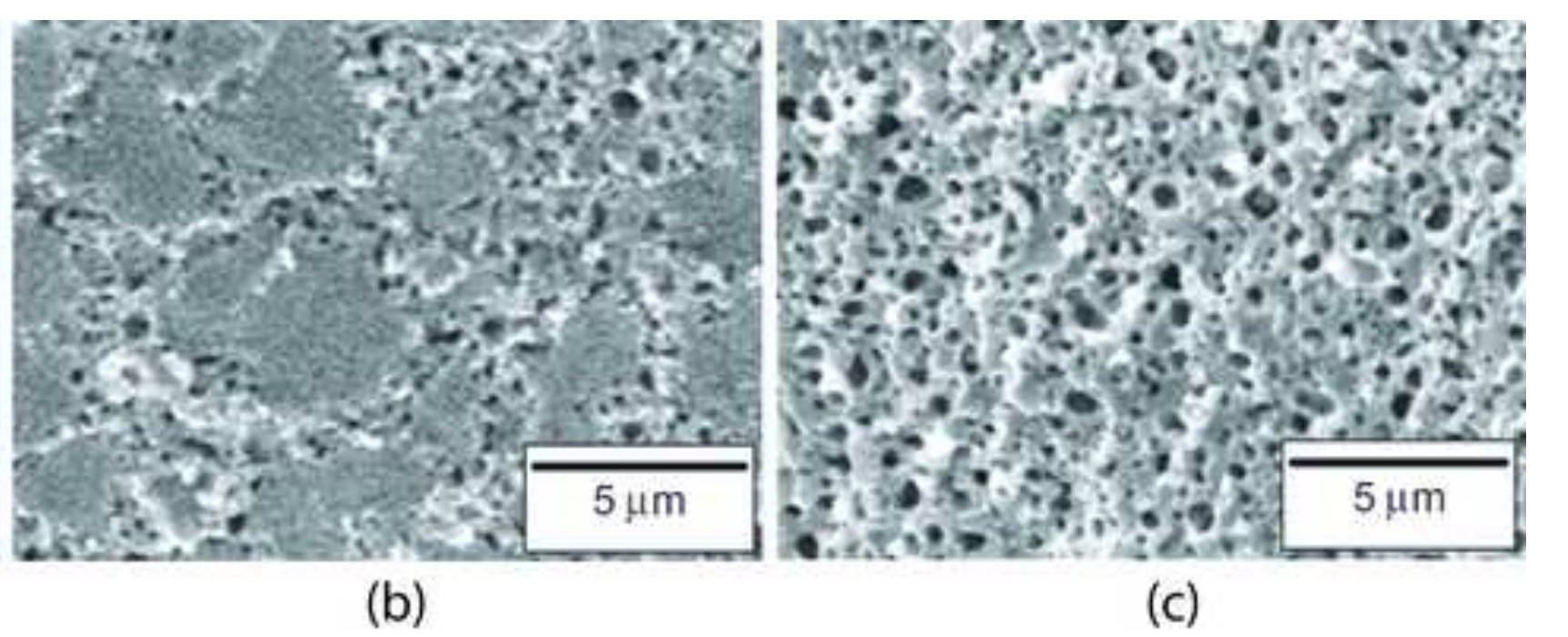

a) (b) (c)

(c)

.

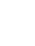

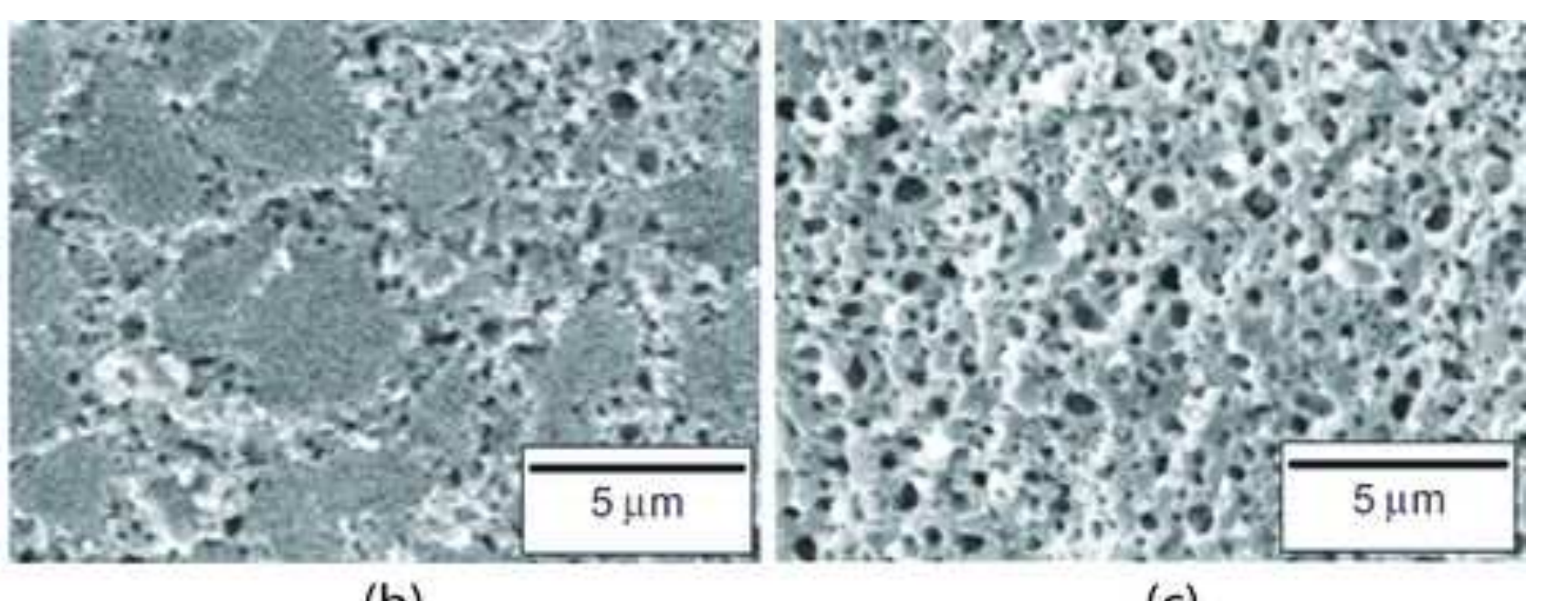

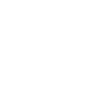

(1)
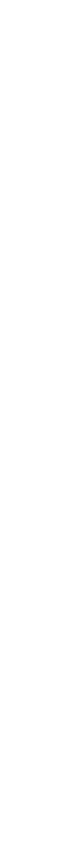
(a)

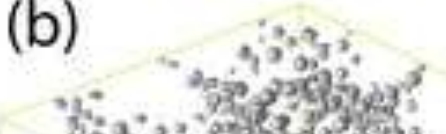

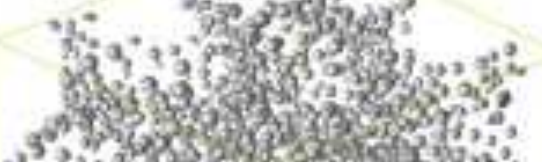

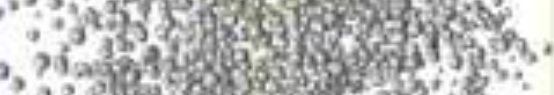
3 ?

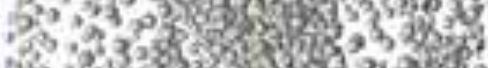

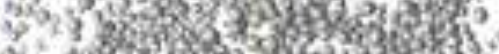

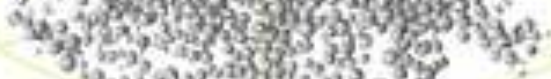

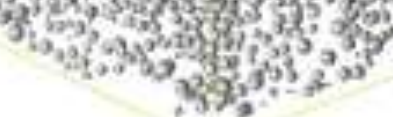

(c)

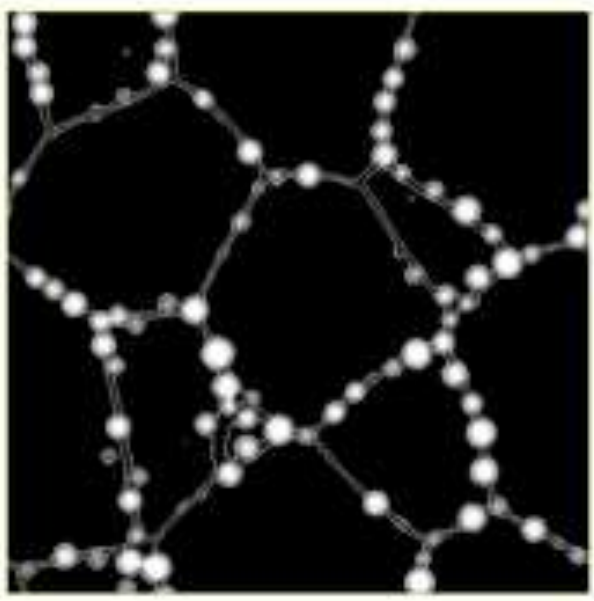

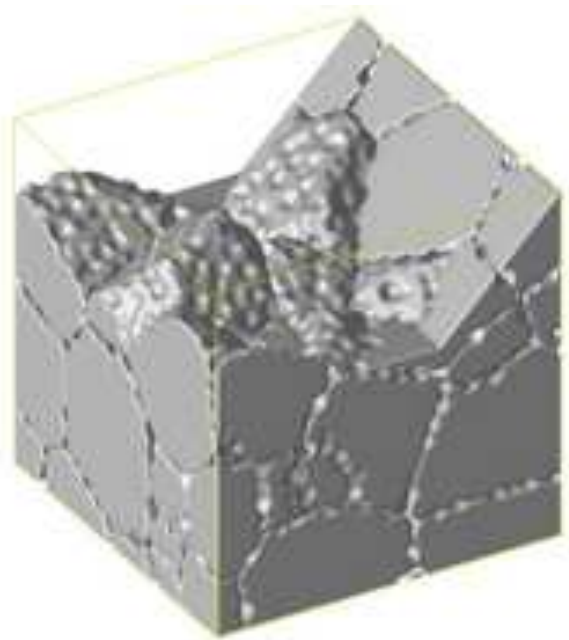
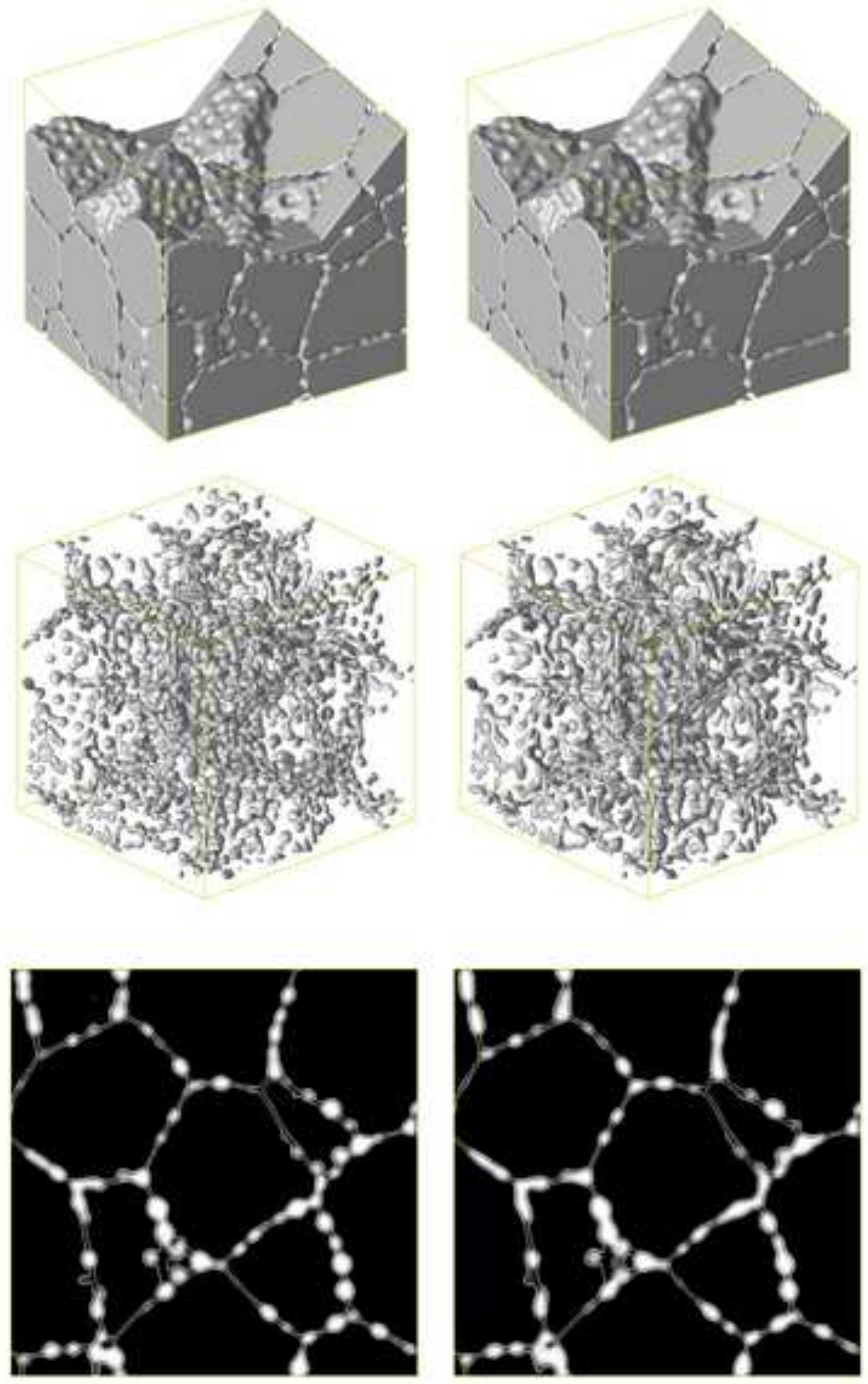

Time 

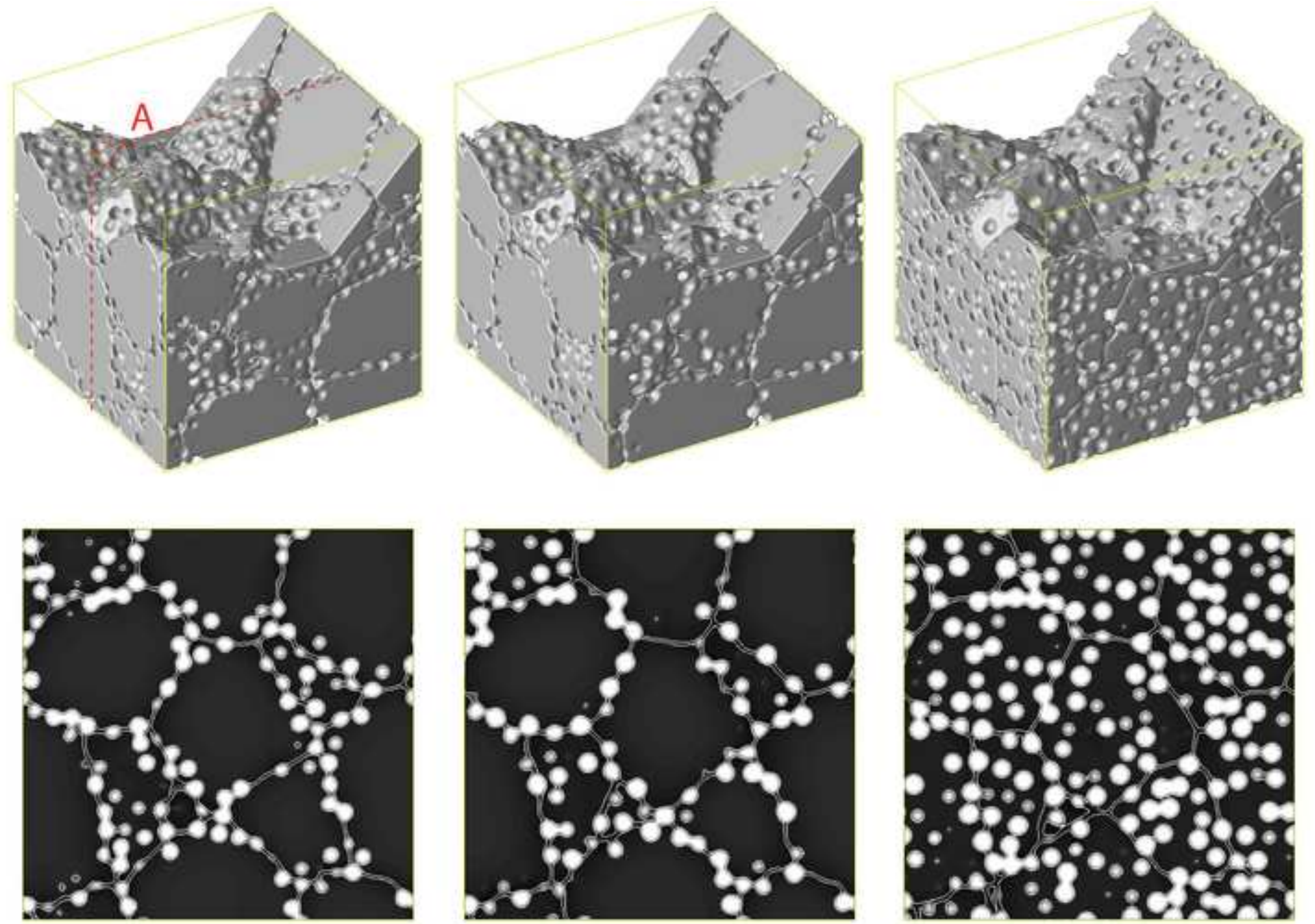

Time 


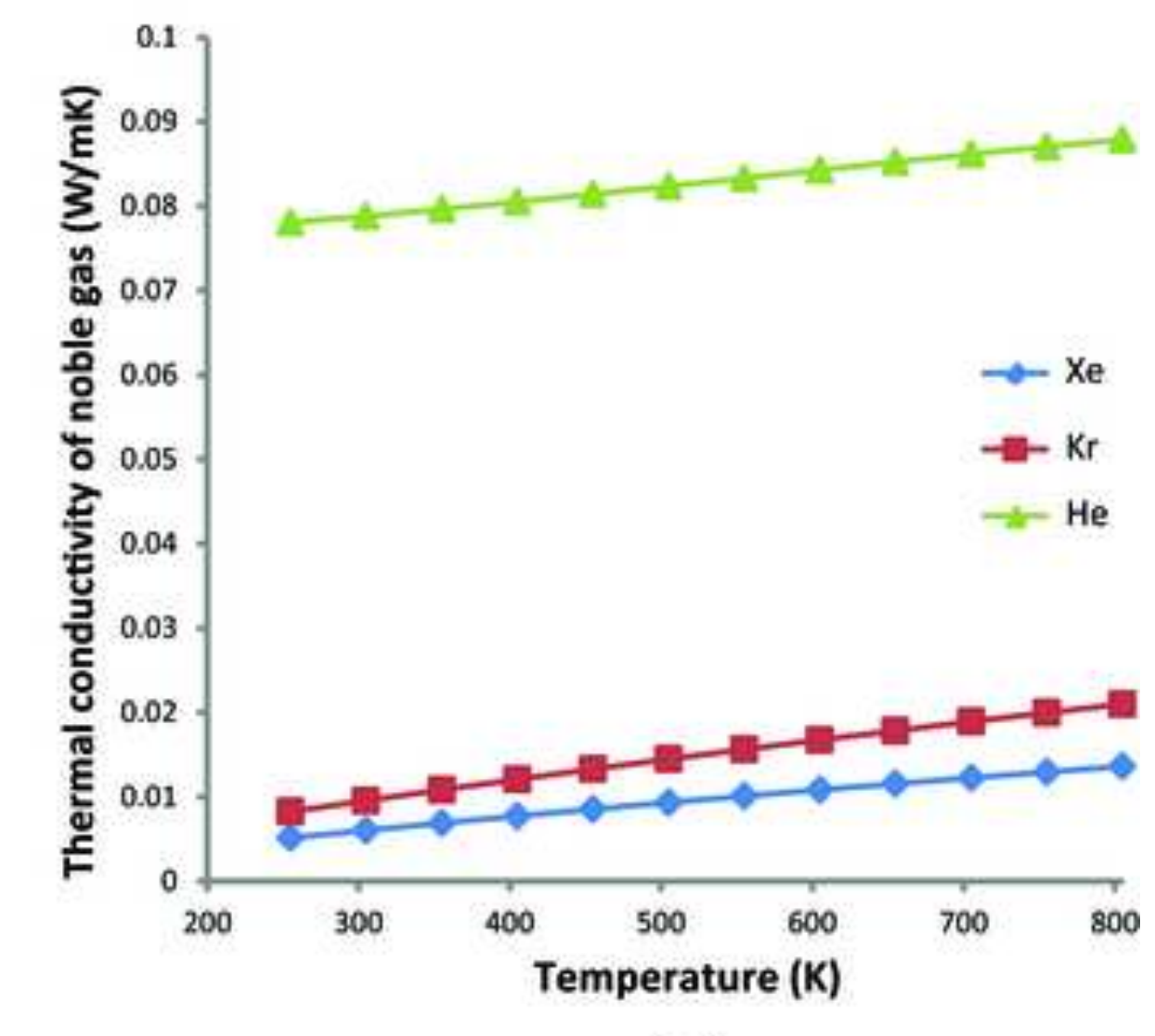

(b)

.

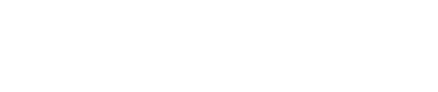

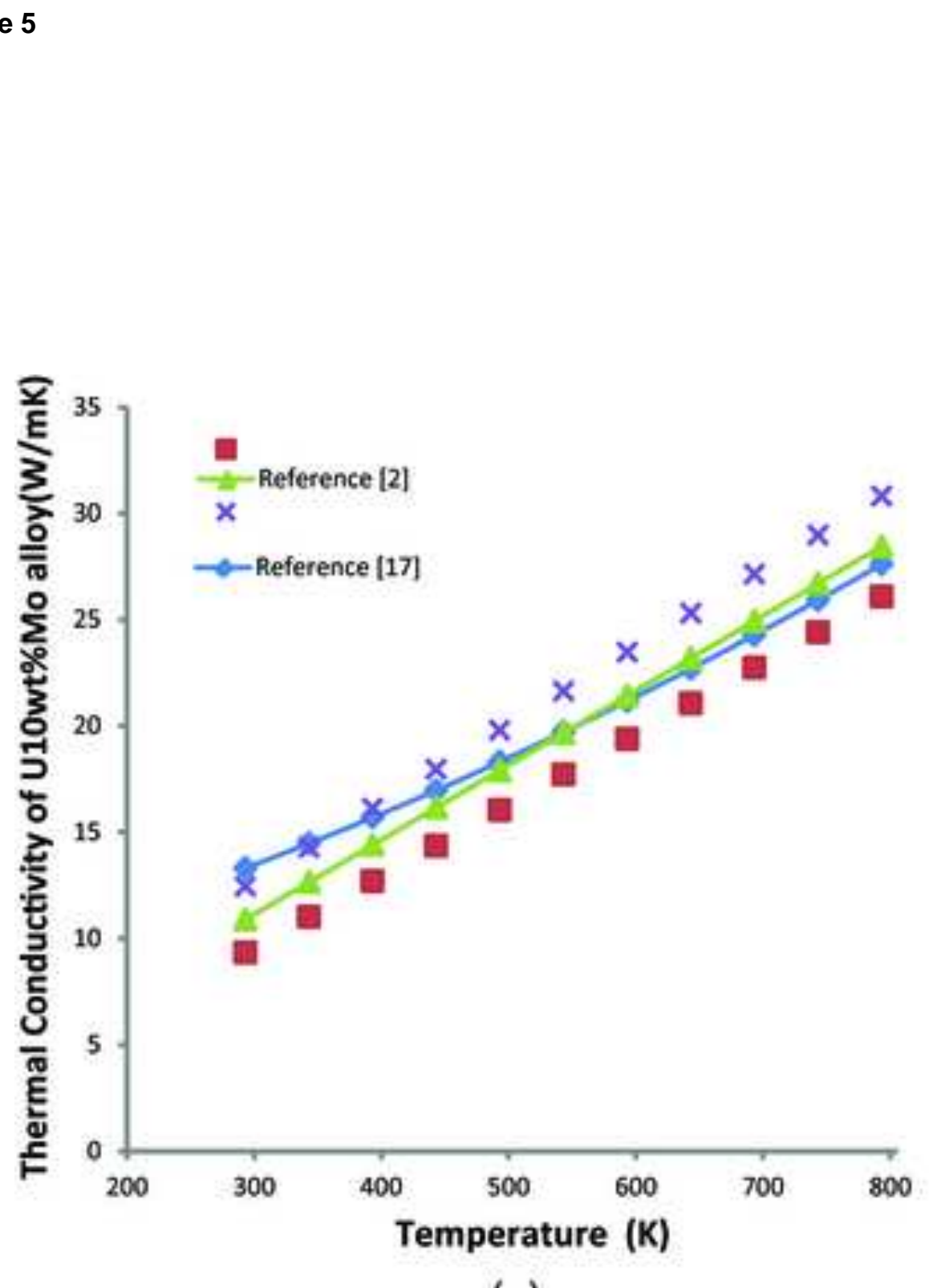

(a)

(a)

\section{Figure}

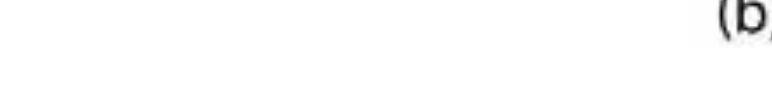




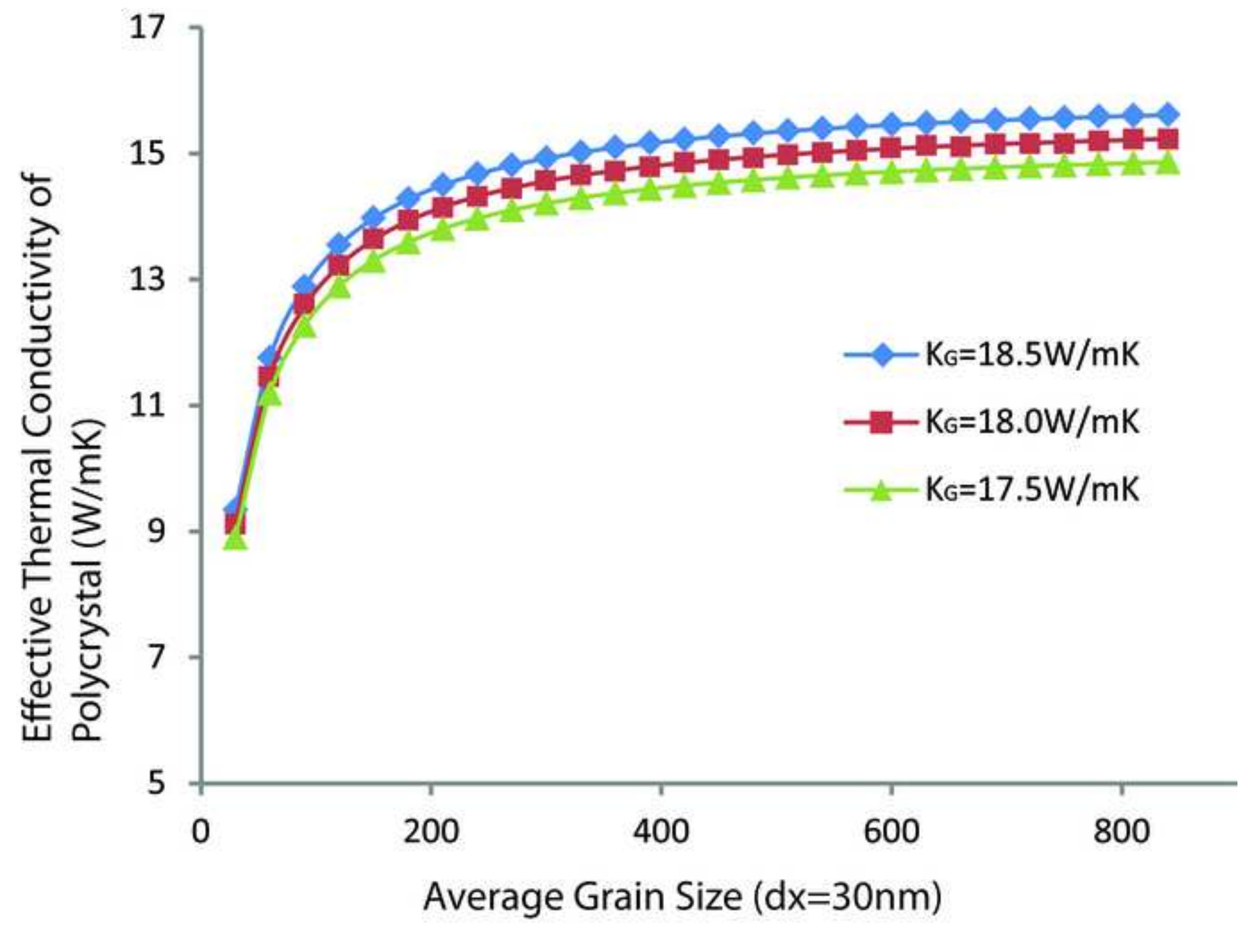
. . .

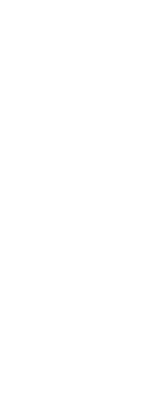

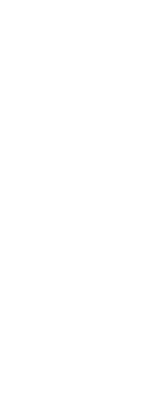
Avera 


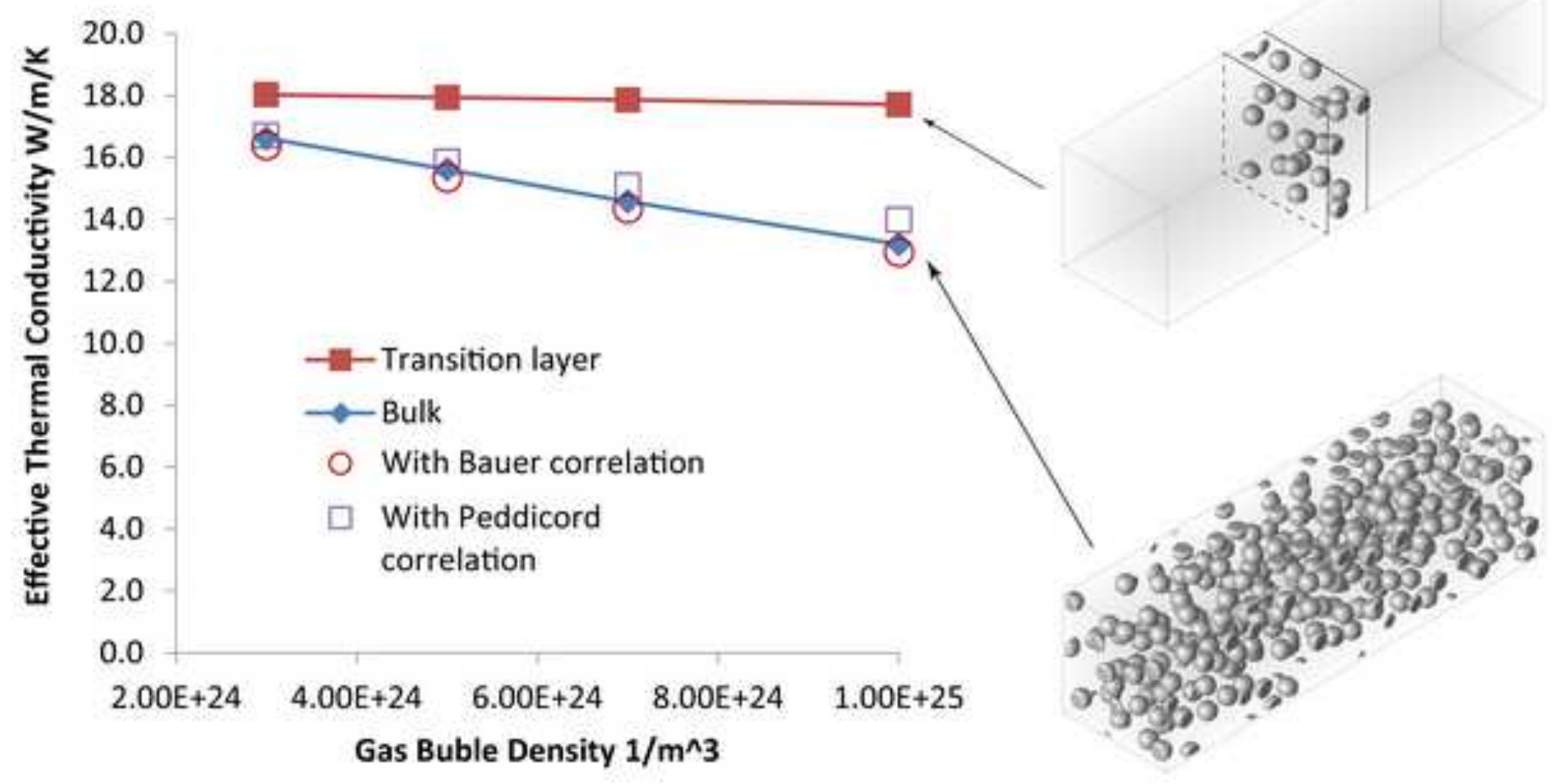




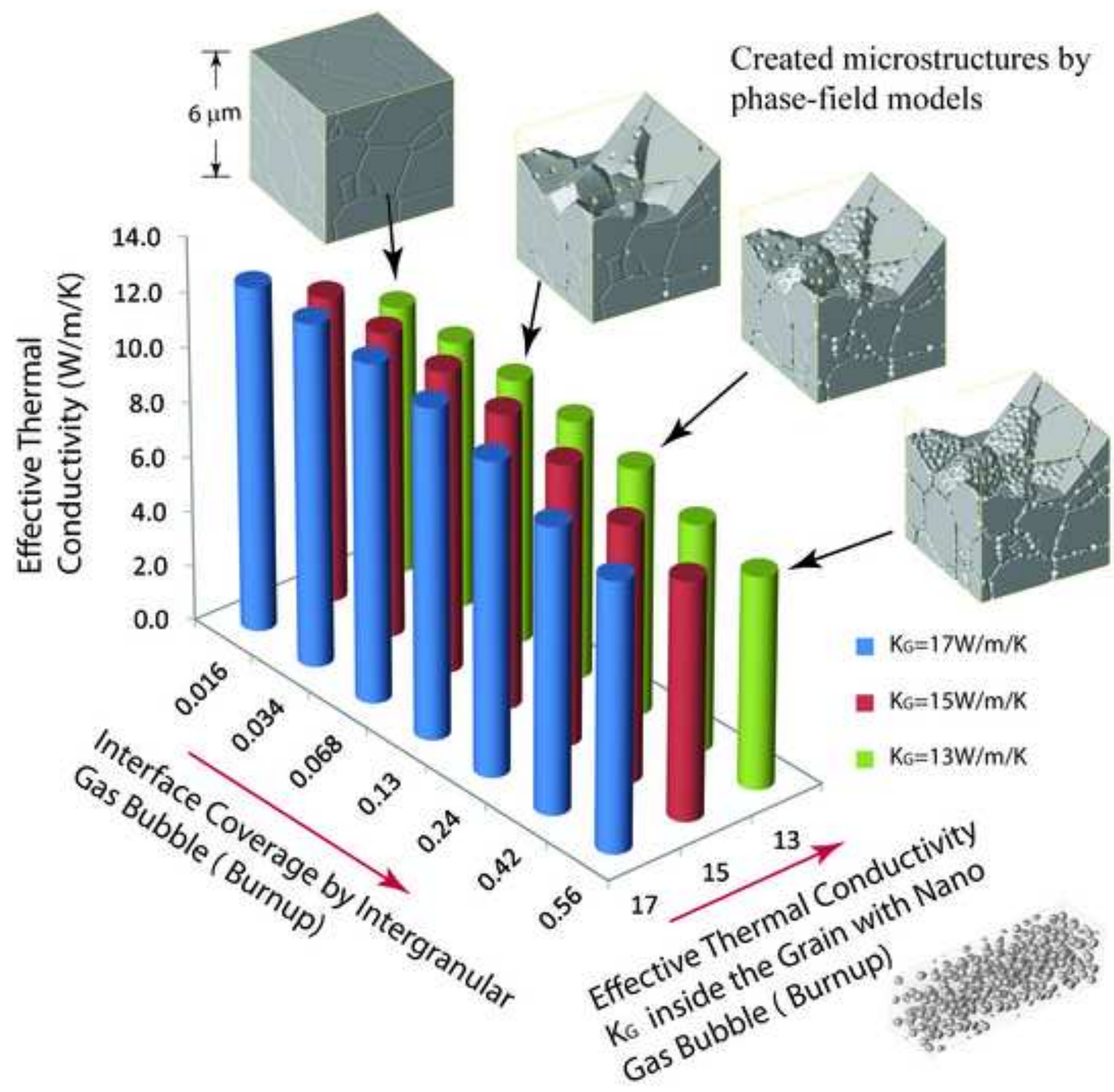




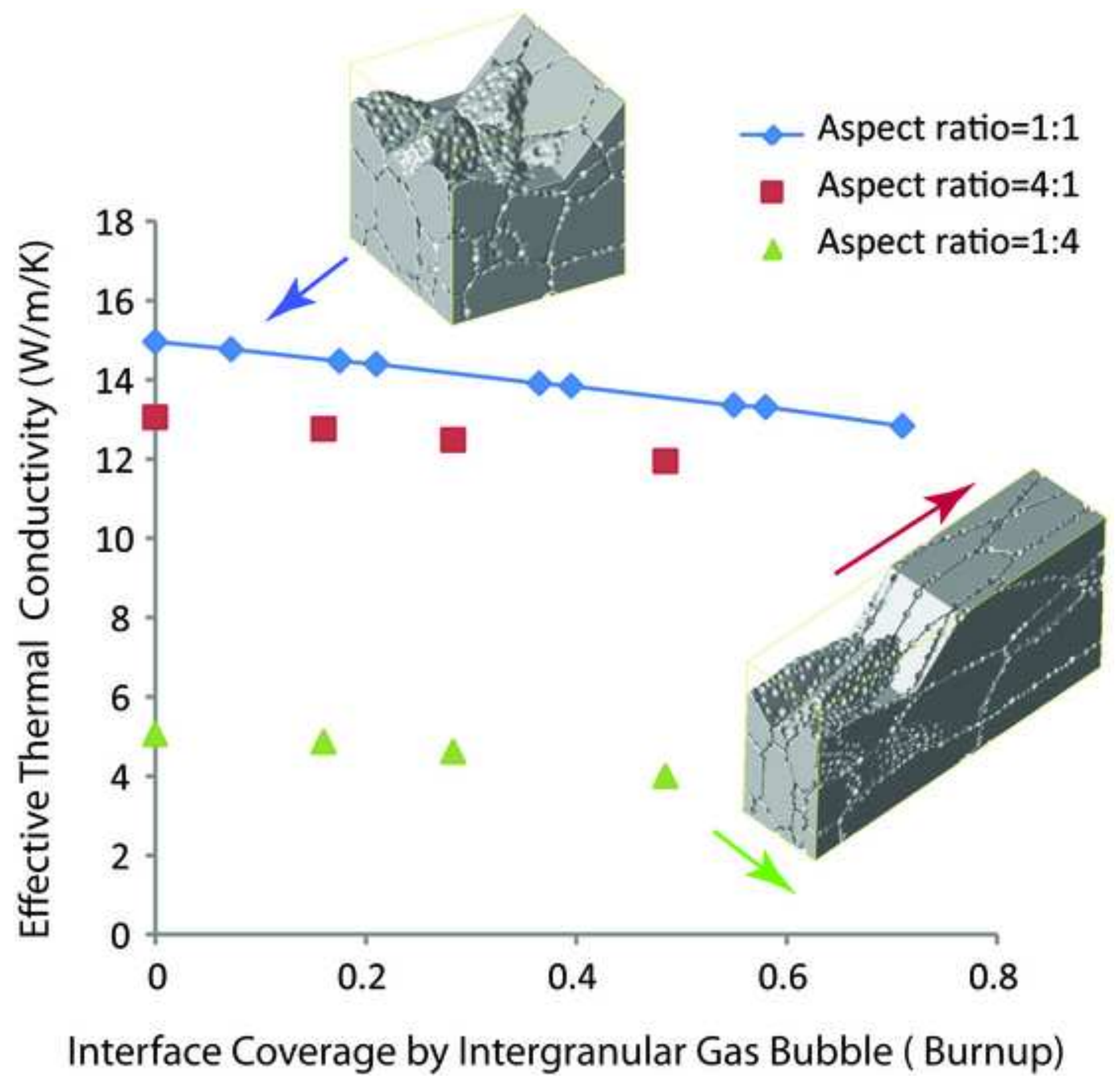

- Aspect ratio $=4: 1$

A Aspect ratio $=1: 4$

Interface Coverage by Intergranular Gas Bubble ( Burnup) 


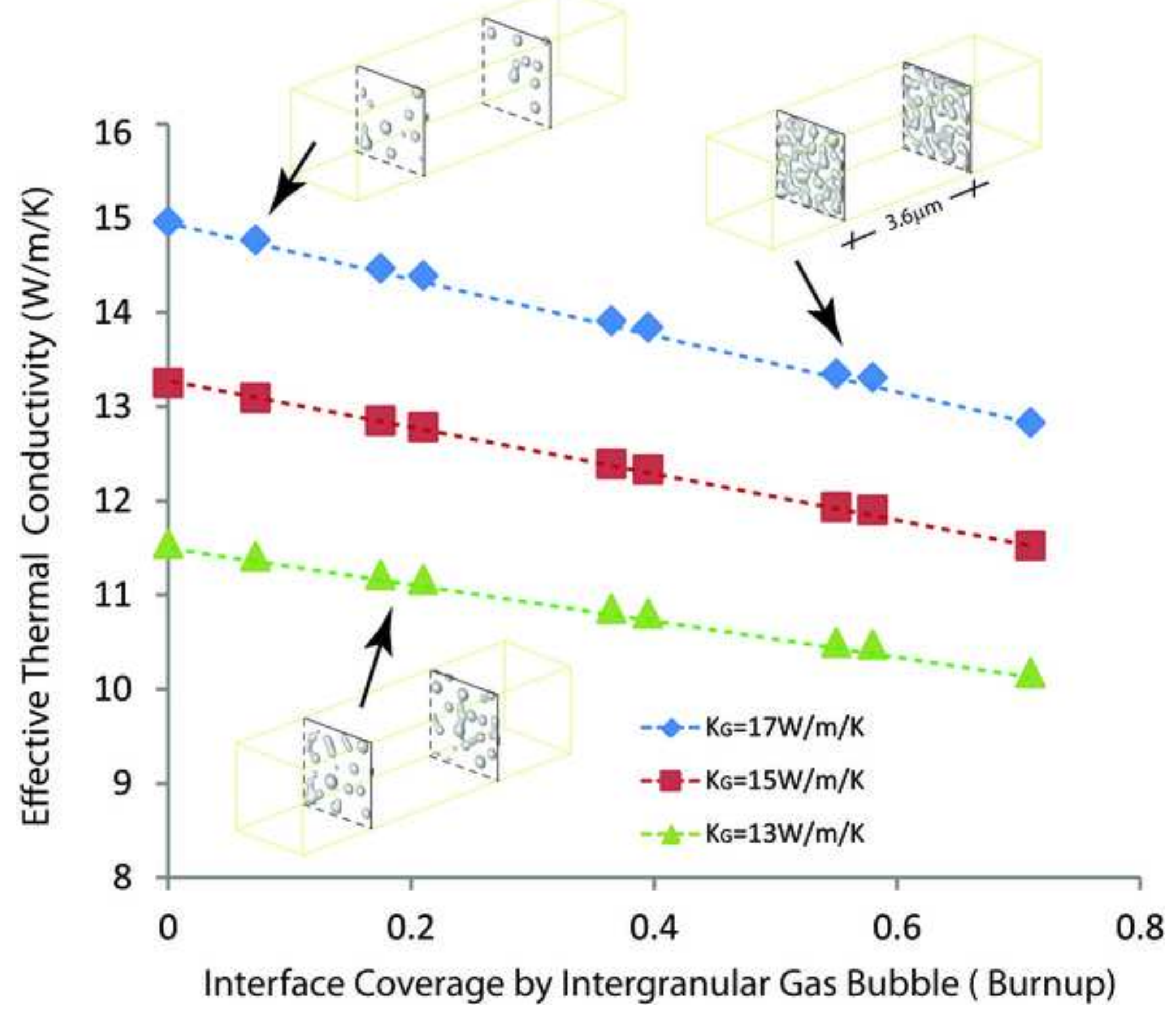




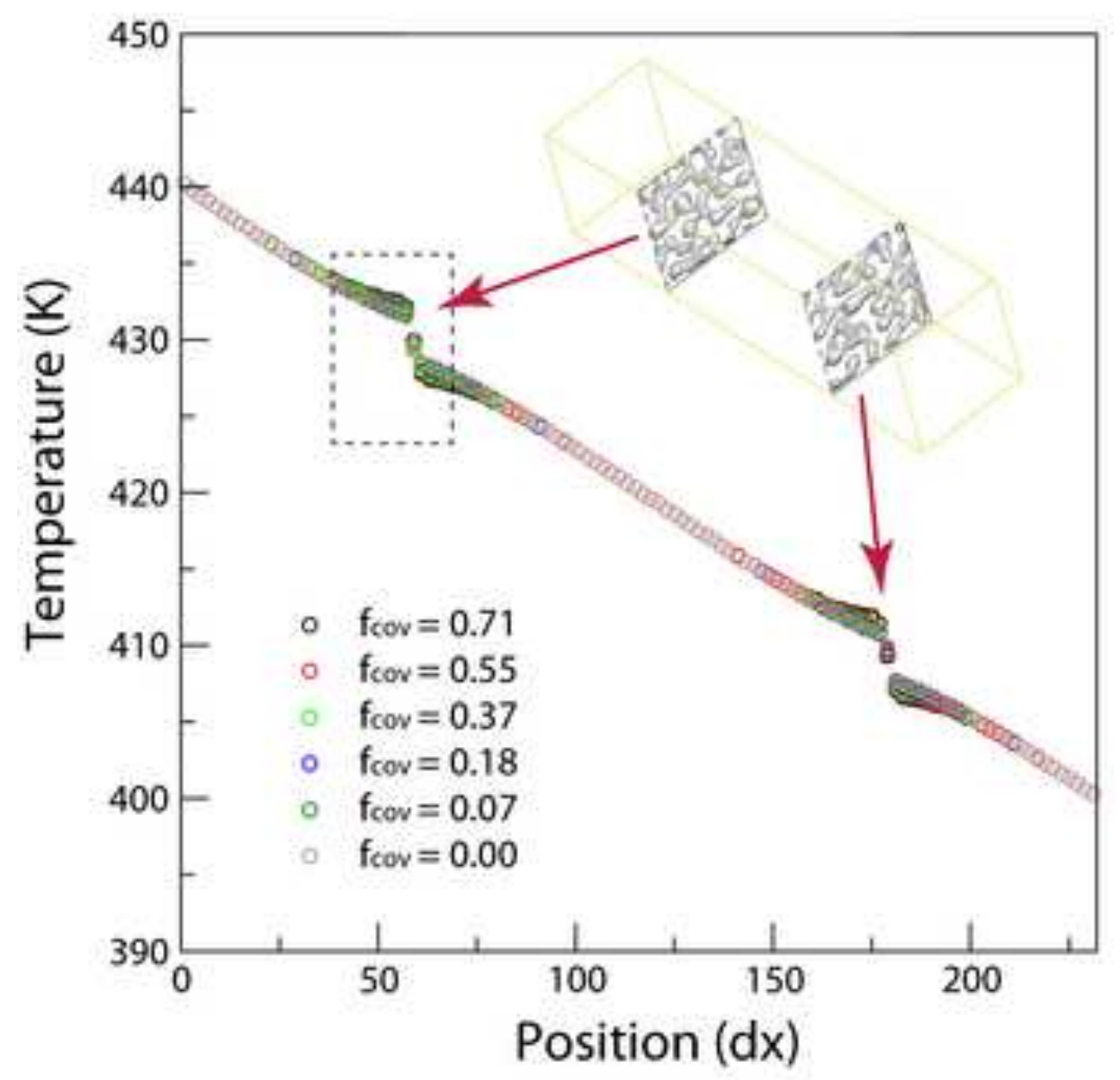

(a)

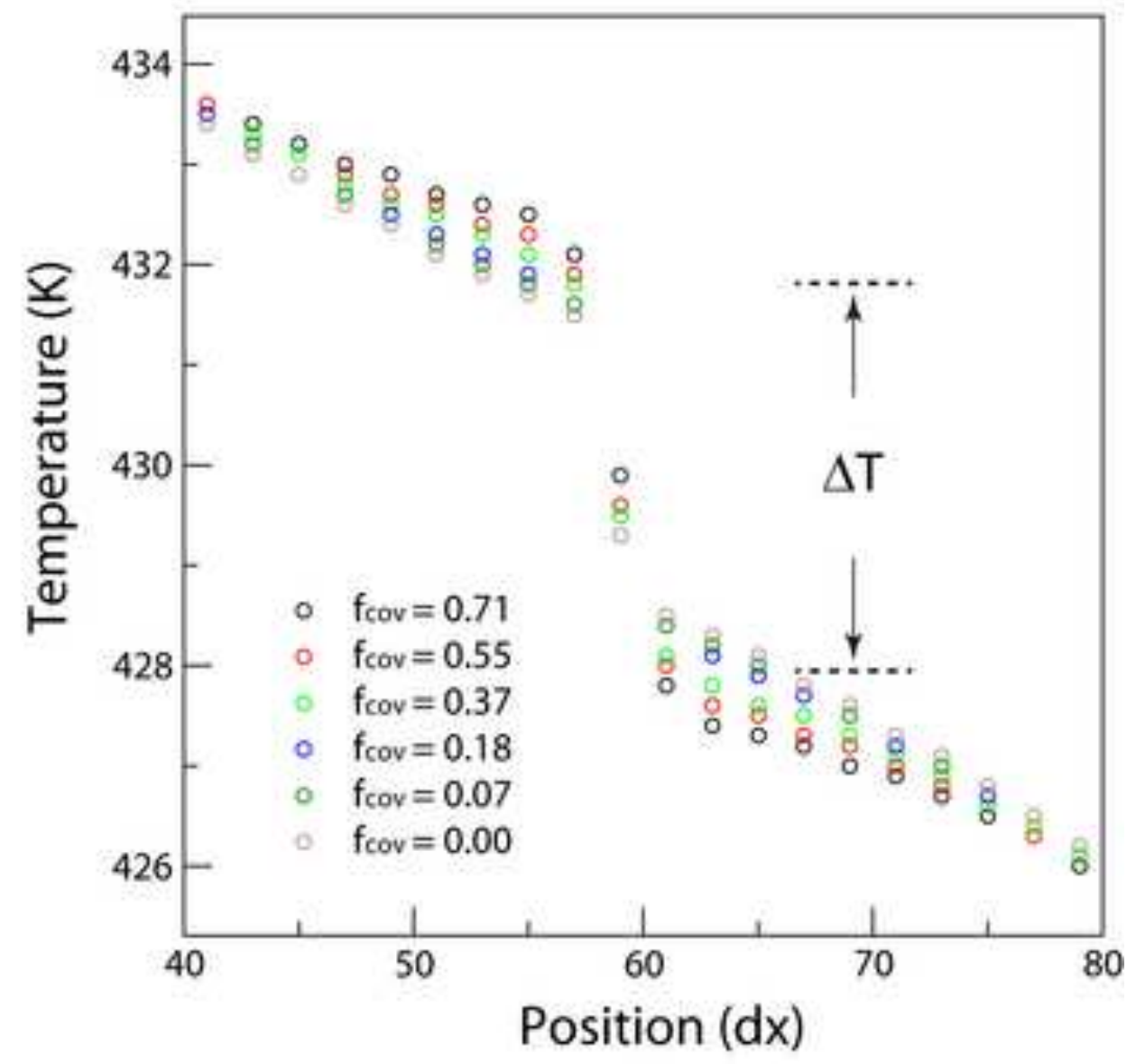

(b) 


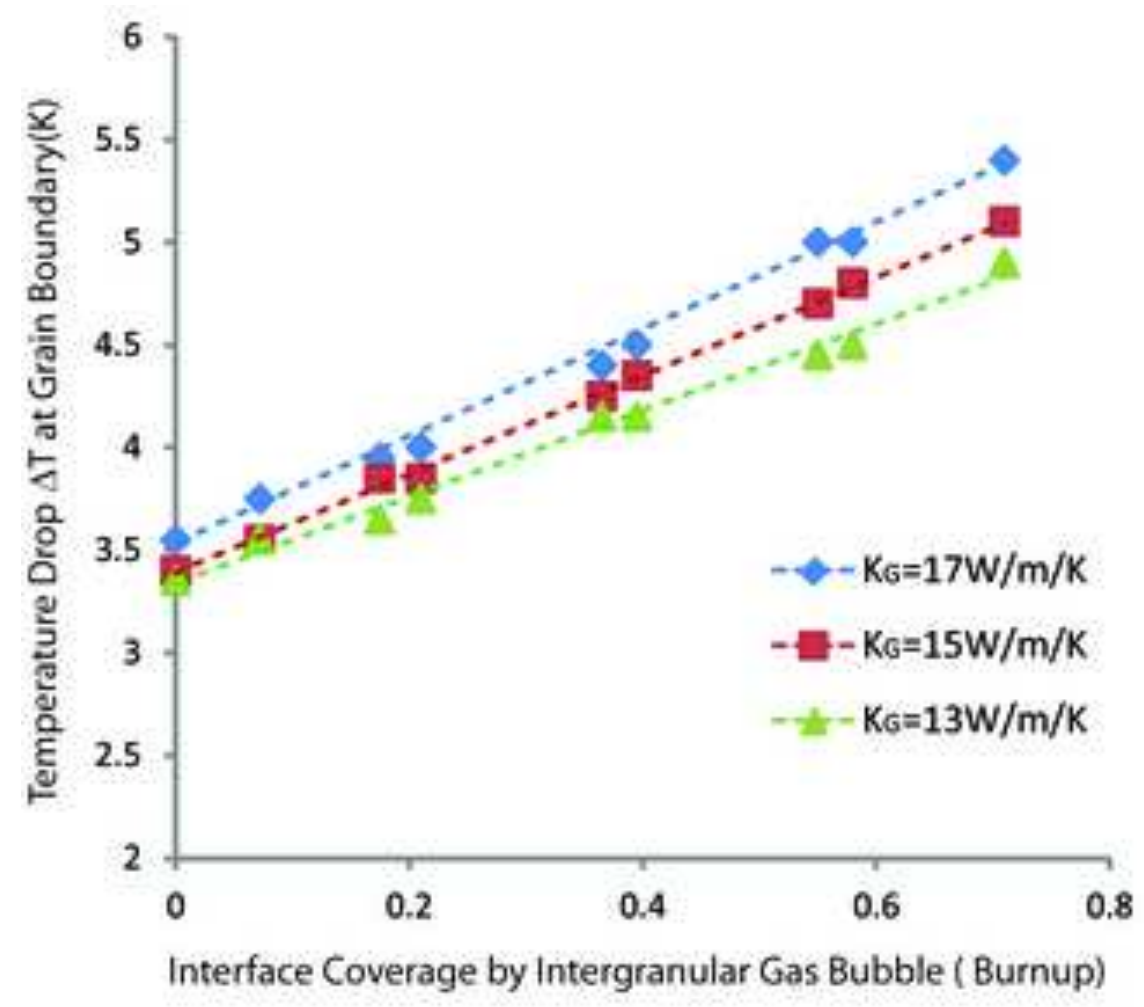

(a)

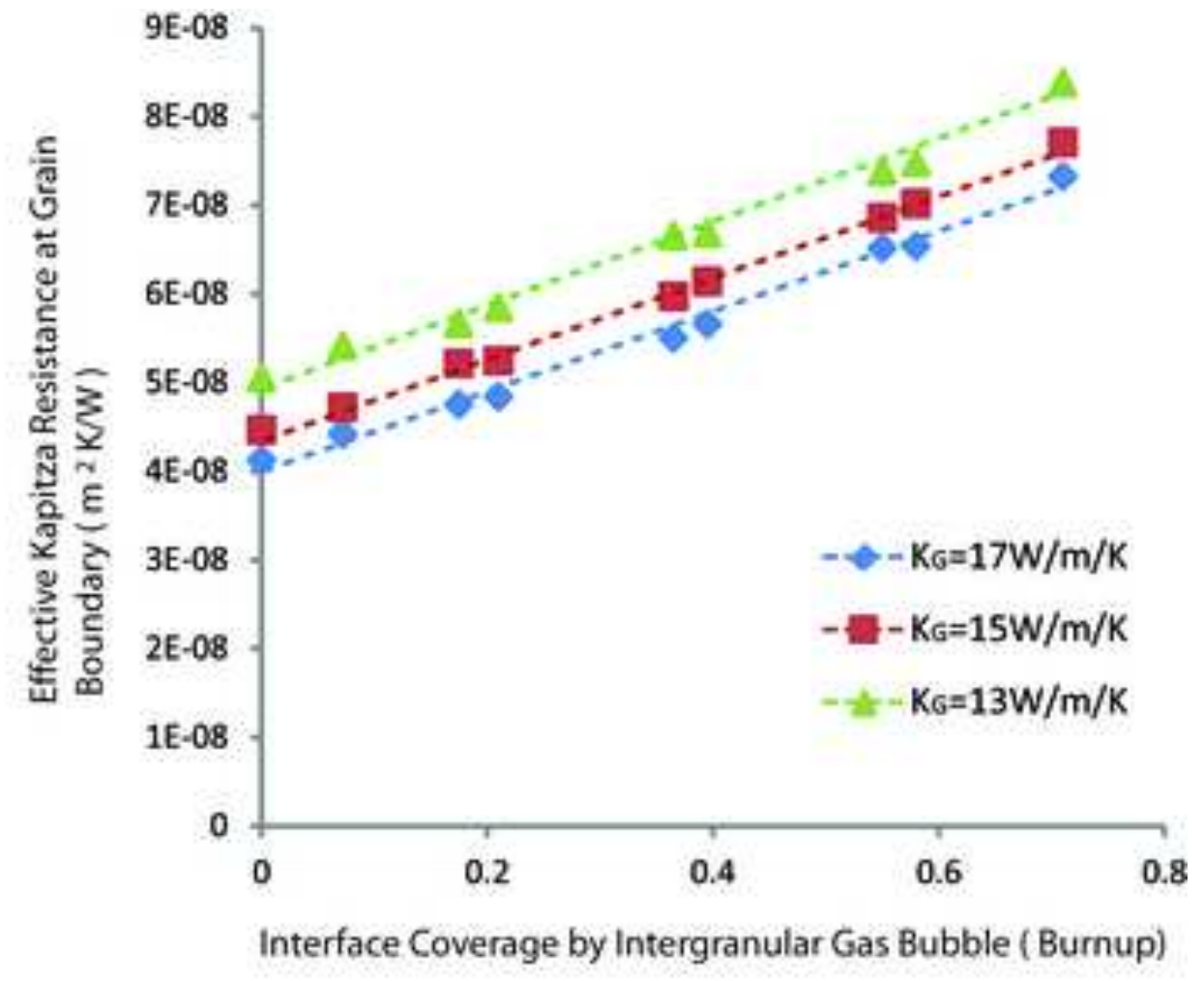

(b) 


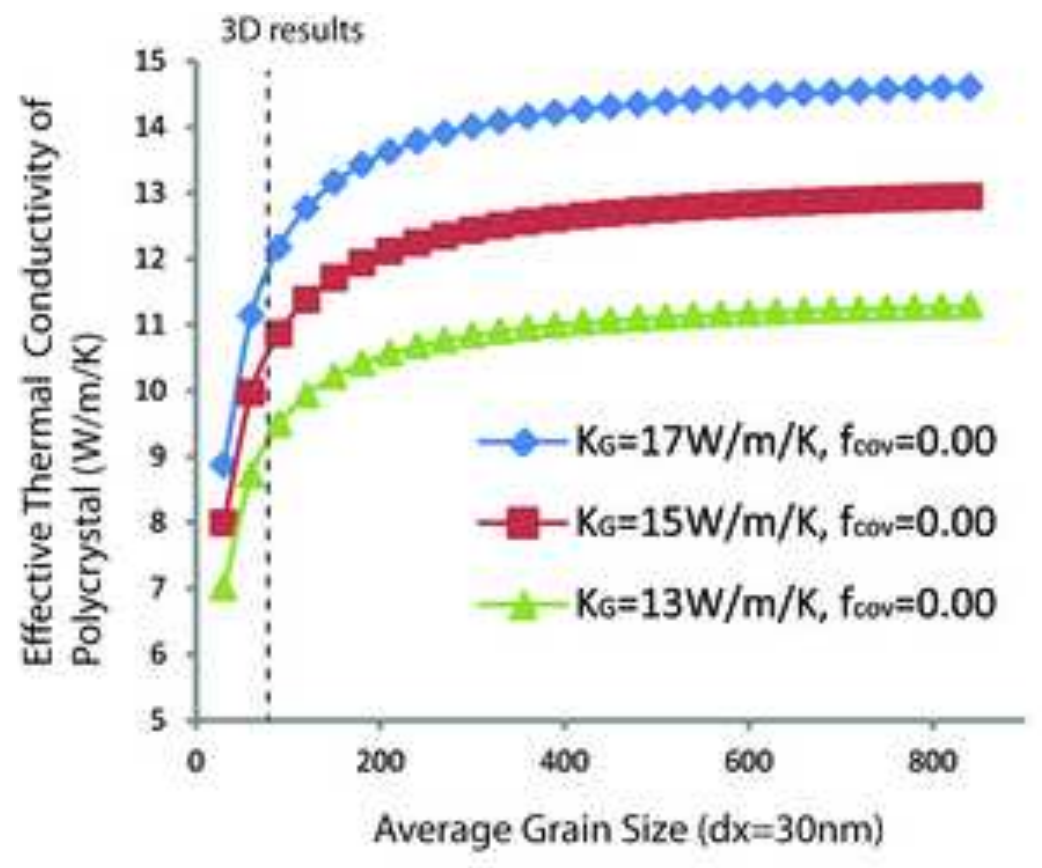

(a)

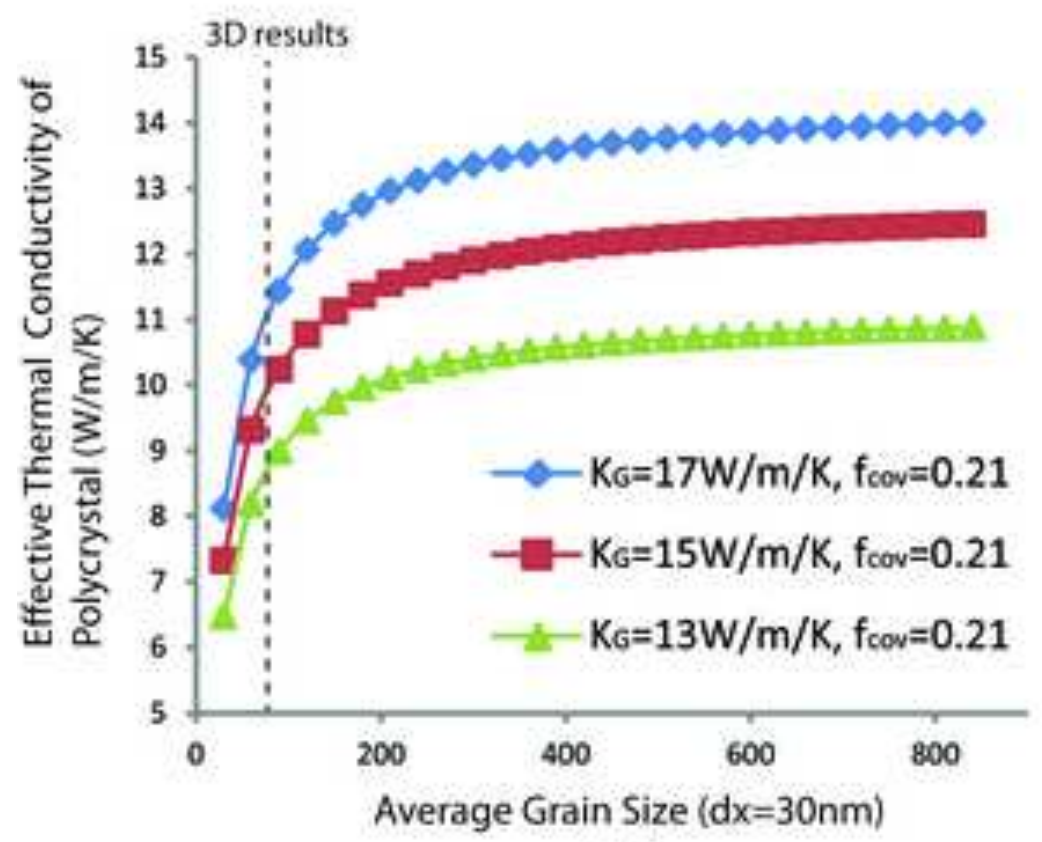

(b)

(b)

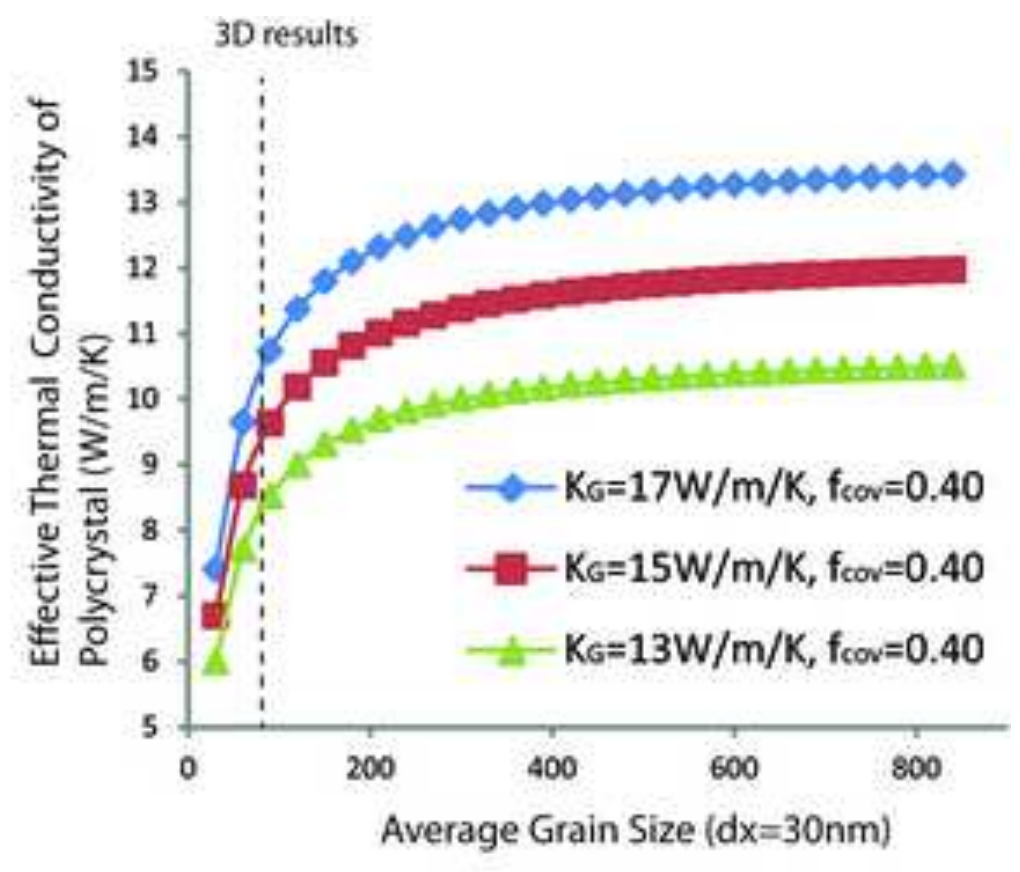

(c)

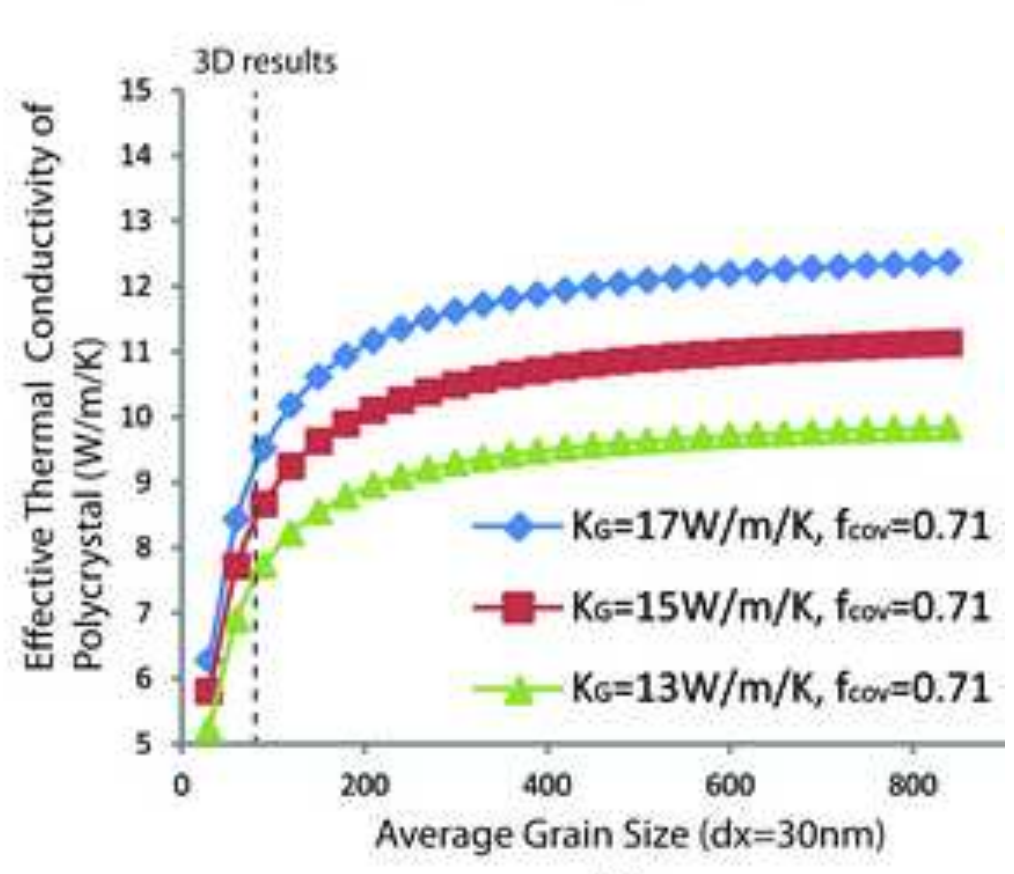

(d)

.

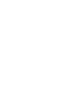




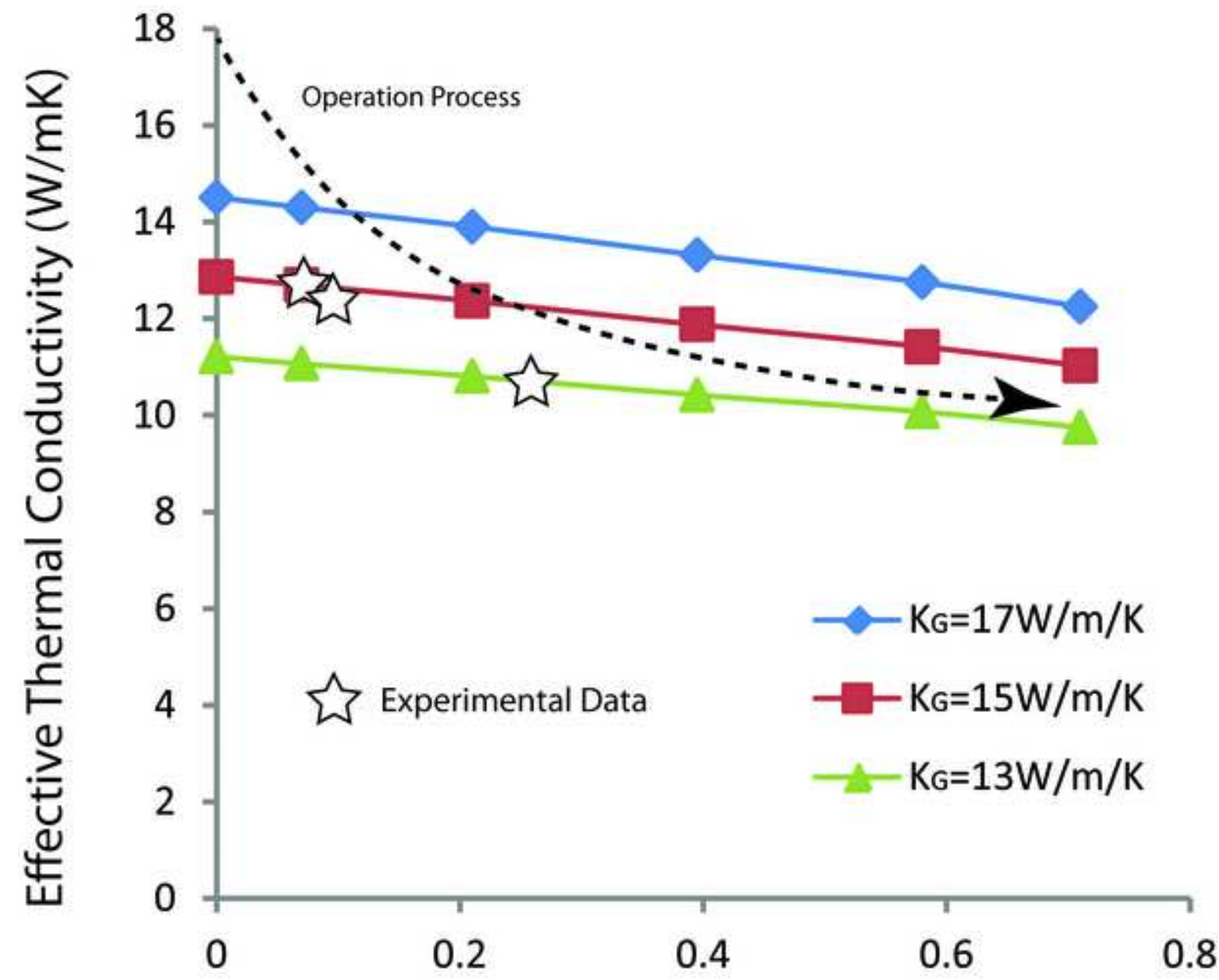

Grain Boundary Coverage by Gas Bubbles 


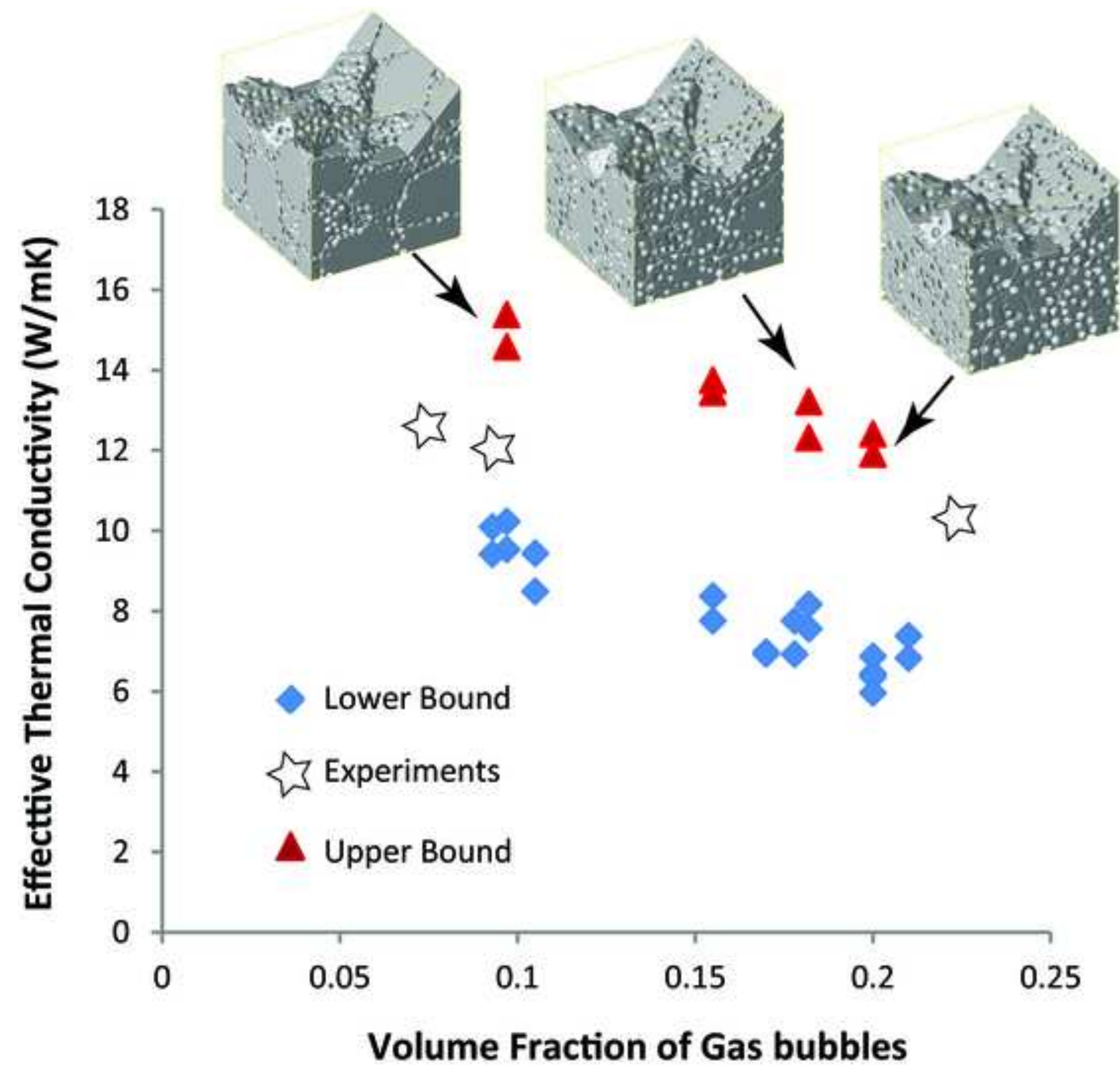

